\title{
HALF-ORDER DIFFERENTIALS ON RIEMANN SURFACES
}

BY

\author{
N. S. HAWLEY and M. SCHIFFER \\ Stanford University, Stanford, Calif., U.S.A. (') \\ Dedicated to Professor R. Nevanlinna on the occasion of his 70th birthday
}

\section{Introduction}

In this paper we wish to exhibit the utility of differentials of half integer order in the theory of Riemann surfaces. We have found that differentials of order $\frac{1}{2}$ and order $-\frac{1}{2}$ have been involved implicitly in numerous earlier investigations, e.g., Poincaré's work on Fuchsian functions and differential equations on Riemann surfaces. But the explicit recognition of these differentials as entities to be studied for their own worth seems to be new. We believe that such a study will have a considerable unifying effect on various aspects of the theory of Riemann surfaces, and we wish to show, by means of examples and applications, how some parts of this theory are clarified and brought together through investigating these half-order differentials.

A strong underlying reason for dealing with half-order differentials comes from the general technique of contour integration; already introduced by Riemann. In the standard theory one integrates a differential (linear) against an Abelian integral (additive function) and uses period relations and the residue theorem to arrive at identities. As we shall demonstrate, one can do an analogous thing by multiplying two differentials of order $\frac{1}{2}$ and using the same techniques of contour integration.

As often happens, when one discovers a new (at least to him) entity and starts looking around to see where it occurs naturally, one is stunned to find so many of its hiding places -and all so near the surface.

Our current point of view concerning the study of Riemann surfaces has evolved from an earlier one in which we introduced the notion of a meromorphic connection in analogy with classical notions in real differential geometry; we now view the theory of connections

(1) This work was supported in part by National Science Foundation grant GP 4069 and Air Force contract AF $49(638) 1345$ at Stanford University. 
on Riemann surfaces as being the theory of first order linear differential equations. The present paper is concerned with the next step-second order linear differential equations. The remarkable difference between first and second order linear differential equations on a closed Riemann surface of genus greater than one is that there exist everywhere regular second order equations, although each first order equation must be singular.

\section{The Schwarzian differential parameter and related connections}

1. In the theory of conformal mappings and univalent functions the following expression plays a central role. If $f(z)$ is an analytic function in the plane domain $D$, the function $[5,8,15,20]$

$$
F(z, \zeta)=\log \frac{f(z)-f(\zeta)}{z-\zeta}
$$

is analytic in the Cartesian product domain $D \times D$ except for logarithmic poles. A necessary and sufficient condition for $f(z)$ to be univalent in $D$ is the regularity of $F(z, \zeta)$ in $D \times D$. Since a linear transformation

$$
f^{*}(z)=\frac{a f(z)+b}{c f(z)+d}
$$

does not affect the univalence of the function, it is to be expected that the corresponding function in two variables $F^{*}(z, \zeta)$ stands in a simple relation to $F(z, \zeta)$. Indeed, we find

$$
F^{*}(z, \zeta)=F(z, \zeta)+\log \frac{a d-b c}{(c f(z)+d)(c f(\zeta)+d)}
$$

Thus it seems useful to define

$$
U(z, \zeta)=\frac{\partial^{2}}{\partial z \partial \zeta} F(z, \zeta)=\frac{f^{\prime}(z) f^{\prime}(\zeta)}{[f(z)-f(\zeta)]^{2}}-\frac{1}{(z-\zeta)^{2}}
$$

which is in view of (3) invariant under linear transformation. Clearly, the univalence of $f(z)$ in $D$ is still equivalent to the regularity of $U(z, \zeta)$ in $D \times D$.

Let $w=f(z)$ and $\omega=f(\zeta)$ and define

$$
[w, \omega ; z, \zeta]=\frac{f^{\prime}(z) f^{\prime}(\zeta)}{[f(z)-f(\zeta)]^{2}}-\frac{1}{(z-\zeta)^{2}}
$$

If we consider $z=g(t), \zeta=g(\tau)$, we can form the analogous expression

$$
[z, \zeta ; t, \tau]=\frac{g^{\prime}(t) g^{\prime}(\tau)}{[g(t)-g(\tau)]^{2}}-\frac{1}{(t-\tau)^{2}}
$$


It is easy to verify that for $w=f\{g(t)\}, \omega=f\{g(\tau)\}$ we have

$$
[w, \omega ; z, \zeta] d z d \zeta+[z, \zeta ; t, \tau] d t d \tau=[w, \omega ; t, \tau] d t d \tau
$$

This additive law for $[w, \omega ; z, \zeta] d z d \zeta$ under composition of mappings is of significant value in the theory of conformal mappings.

If we identify the two arguments in $U(z, \zeta)$, we obtain

$$
U(z, z)=[w, w ; z, z]=-\frac{1}{6}\left[\left(\frac{f^{\prime \prime}(z)}{f^{\prime}(z)}\right)^{\prime}-\frac{1}{2}\left(\frac{f^{\prime \prime}(z)}{f^{\prime}(z)}\right)^{2}\right] .
$$

We are thus led in a natural way to the Schwarz differential parameter

$$
\{w, z\}=\left(\frac{f^{\prime \prime}(z)}{f^{\prime}(z)}\right)^{\prime}-\frac{1}{2}\left(\frac{f^{\prime \prime}(z)}{f^{\prime}(z)}\right)^{2}
$$

in terms of which (8) can be expressed as

$$
U(z, z)=[w, w ; z, z]=-\frac{1}{6}\{w, z\}
$$

From the preceding properties of $U(z, \zeta)$ follow then the well-known properties of the Schwarzian differential parameter:

(a) $\{w, z\}$ is invariant under a linear transformation of $w$.

(b) Under the composition $w=f(z)$ and $z=g(t)$ we have

$$
\{w, z\} d z^{2}+\{z, t\} d t^{2}=\{w, t\} d t^{2}
$$

From (a) follows that for the linear transformation

$$
z=\frac{\alpha t+\beta}{\gamma t+\delta}
$$

holds identically $\{z, t\}=0$. Hence we infer from (b) in this case:

$$
\{w, t\} d t^{2}=\{w, z\} d z^{2}
$$

that is, the Schwarzian differential parameter transforms like a quadratic differential under a linear transformation of the independent variable.

Finally, let in (10) $t=w$ and use the fact that in this case again $\{w, t\}=0$. Thus

$$
\{w, z\} d z^{2}=-\{z, w\} d w^{2}
$$

which determines the Schwarzian differential parameter of inverse functions. 
We may consider $w$ as a function on the domain $D$ and the independent variable $z$ as local coordinate. Then the mapping $z=g(t)$ can be conceived as a change of coordinates and the transformation law (10) shows that the Schwarzian differential parameter $\{w, z\}$ transforms under such change of coordinates according to a linear inhomogeneous law of transformations. The expression behaves almost like a quadratic differential; however, we have to add the inhomogeneity $\{z, t\} d t^{2}$ which does not depend on the function $w$ considered, but only on the transformation law from $z$ to $t$. We may call $\{w, z\}$ a connection in analogy to the corresponding concept in differential geometry [18].

2. Naturally we are now led to consider connections analogous to the Schwarzian differential parameter on Riemann surfaces. We ask for quantities $S_{z}$ defined in terms of the local uniformizer $z$, which transform under a change of uniformizer $z=g(t)$ according to the law

$$
S_{t}(t) d t^{2}=S_{z}(z) d z^{2}+\{z, t\} d t^{2}
$$

Clearly, it is enough to construct on a given Riemann surface only one such Schwarzian connection. For, if $S_{z}$ and $S_{z}$ are two such expressions with the law of transformation (14), their difference would be a quadratic differential on the surface and this class is well known and completely understood.

It is now remarkable that on every Riemann surface $\mathfrak{R}$ there does indeed exist a regular analytic Schwarzian connection $S$. In order to construct it, we introduce the Abelian integral of the third kind $[10,14,22] w(p ; r, s)$ which is analytic in $p$, has logarithmic poles with residues +1 and -1 , respectively, at the two given points $r \in \Re$ and $s \in \Re$ and which is normalized to have the periods zero with respect to the cross cuts $\mathfrak{A}_{\nu}$ of a canonical cut system $\left\{\mathfrak{A}_{\nu}, \mathfrak{B}_{\nu}\right\}$. The analytic dependence of the Abelian integral so defined upon its parameters is best understood by means of the fundamental theorem that for every quadruple $p, q ; r, s$ on $\mathfrak{R}$ the combination

$$
W(p, q ; r, s)=w(p ; r, s)-w(q ; r, s)
$$

is symmetric in the pair $p, q$ of arguments and $r, s$ of parameters. In particular, we see that

$$
\frac{\partial^{2} w(p ; r, s)}{\partial p \partial r}=\frac{\partial^{2} w(r ; p, q)}{\partial r \partial p}=\frac{\partial^{2} W(p, q ; r, s)}{\partial p \partial r}
$$

depends analytically on $p$ and $r$, is symmetric in $p$ and $r$ and is independent of $s$ and $q$. This expression has a singularity if $p=r$, and to study it we introduce a local uniformizer $z$ such that $p$ has the coordinate $z$ and $r$ has the coordinate $\zeta$. We then find that 


$$
\frac{\partial^{2} W(p, q ; r, s)}{\partial z \partial \zeta}=\frac{1}{(z-\zeta)^{2}}+l_{z}(z, \zeta)
$$

where $l_{z}(z, \zeta)$ is symmetric in both variables and regular analytic in the uniformizer neighborhood. If we change uniformizers by the analytic relations $z=g(t), \zeta=g(\tau)$, we find by use of (6)

$$
l_{t}(t, \tau) d t d \tau=l_{z}(z, \zeta) d z d \zeta+[z, \zeta ; t, \tau] d t d \tau
$$

Let us then define the expression

$$
S_{z}(z)=-6 l_{z}(z, z)=\lim _{\zeta \rightarrow z}\left[-6\left(\frac{\partial^{2} W(p, q ; r, s)}{\partial z \partial \zeta}-\frac{1}{(z-\zeta)^{2}}\right)\right] .
$$

This is an analytic function in each uniformizer neigborhood which depends, however, upon the choice of uniformizer. In view of $\left(8^{\prime}\right)$ and (18) we have

$$
S_{t}(t) d t^{2}=S_{z}(z) d z^{2}+\{z, t\} d t^{2}
$$

A comparison of this transformation law with (14) shows that $S$ satisfies the proper transformation rule of a connection.

We obtain all Schwarzian connections on $\mathfrak{R}$ by adding to the expression $S_{z}(z)$ constructed explicitly any quadratic differential on $\Re$. However, we may construct another Schwarzian connection in a different way and arrive at a remarkable identity. Let $\varphi(p)$ be the analytic function which is defined on the universal covering surface $\mathfrak{R}_{u}$ of $\mathfrak{R}$ and maps $\Re_{u}$ onto the unit disk. It is well known that the $\varphi(p)$ is polymorphic on $\mathfrak{R}$, that is, at points of $\mathfrak{R}_{u}$ over the same point of $\mathfrak{R}$ the function $\varphi(p)$ has different determinations which are related by a linear transformation. Thus, if $\hat{\varphi}(p)$ and $\varphi(p)$ are two such determinations, then

$$
\hat{\varphi}(p)=L[\varphi(p)]
$$

where $L$ transforms the unit disk onto itself. If we introduce a local uniformizer $z$ at $p$, we see that in view of (21)

$$
\{\varphi, z\}=\{\hat{\varphi}, z\}
$$

that is, the Schwarzian differential parameter of the polymorphic function $\varphi(p)$ is the same for all branches of this function; it is single-valued and analytic in each uniformizer neighborhood on $\mathfrak{R}$. It depends, however, on the choice of the uniformizer; if we replace $z$ by $t$ through the analytic relation $z=g(t)$, we find by (10)

$$
\{\varphi, t\} d t^{2}=\{\varphi, z\} d z^{2}+\{z, t\} d t^{2}
$$


Thus $\{\varphi, z\}$ has the same transformation law of a Schwarzian connection as $S_{z}(z)$. Hence we have

$$
\{\varphi, z\}=-6 l_{z}(z, z)+Q(z)
$$

where $Q(z)$ is a regular quadratic differential on $\Re$.

Since the Abelian integrals and quadratic differentials of a surface $\Re$ are of a more elementary character than the uniformizing transcendatal function $\varphi(z)$, we may consider (24) as a useful differential equation for $\varphi(z)$ in terms of the easier accessible expressions $l_{z}(z, z)$ and $Q(z)$.

\section{Schwarz' differential equation and half-order differentials}

1. We return to the case of planar domains $D$. Suppose that a function $S(z)$ is given in $D$ and that we wish to find the solution of the differential equation of the third order in $w(z)$,

$$
\{w, z\}=S(z)
$$

Schwarz showed that the solution of this nonlinear differential equation can be reduced to the simpler problem of finding two independent solutions of the linear second order equation

$$
u^{\prime \prime}(z)+\frac{1}{2} S(z) u(z)=0 \text {. }
$$

Indeed, if $u_{1}(z)$ and $u_{2}(z)$ are independent solutions of (2), their Wronskian

$$
u_{1}(z) u_{2}^{\prime}(z)-u_{2}(z) u_{2}^{\prime}(z)=W(z)
$$

will be not identically zero. On the other hand, we see from (2) at once that $W(z)$ must be a constant; thus we may choose $W(z) \equiv 1$. In any case, as a simple calculation shows, the ratio

$$
w(z)=\frac{u_{1}(z)}{u_{2}(z)}
$$

will satisfy the differential equation (1).

Let us now change the independent variable by a conformal mapping $z=g(t)$ and refer to the independent variable $t$ in a domain $\Delta$. Clearly, $w=w[g(t)]$ is defined in $\Delta$ by composition and we have

$$
\{w, t\} d t^{2}=S(z) d z^{2}+\{z, t\} d t^{2}
$$

To find $w$ as a function of $t$, we might also consider the corresponding linear second order equation

$$
v^{\prime \prime}(t)+\frac{1}{2}\{w, t\} v(t)=0
$$

and express $w$ as the ratio of two independent solutions of this equation. 
However, the natural question arises whether there is any relation between the solutions $u_{\nu}(z)$ of $(2)$ and $v_{\nu}(t)$ of (6). An easy calculation shows that

$$
v_{\nu}(t)=u_{\nu}(g(t))\left[g^{\prime}(t)\right]^{-\frac{t}{2}}, \quad \nu=1,2
$$

yields a system of two independent solutions of (6) whose Wronskian has also the value 1 .

We have thus the remarkable fact: If $S$ transforms like a Schwarzian connection

$$
S_{t}(t) d t^{2}=S_{2}(z) d z^{2}+\{z, t\} d t^{2}
$$

the solutions of the differential equation

$$
u^{\prime \prime}(z)+\frac{1}{2} S_{z}(z) u(z)=0
$$

transform like differentials of order $-\frac{1}{2}$, i.e.,

$$
u_{t}(t) d t^{-\frac{1}{2}}=u_{z}(z) d z^{-\frac{1}{2}} \text {. }
$$

2. To show the usefulness of this covariance of the auxiliary functions $u_{p}(z)$, we rederive an interesting theorem of Nehari [13] which connects the univalence of a function $f(z)$ analytic in the unit disk with the growth of its Schwarzian differential parameter $S(z)=\{f, z\}$.

Suppose that $f(z)$ is not univalent in $|z|<1$. There would be two different points in the disk, say $a$ and $b$, such that $f(a)=f(b)$. By a linear transformation of the independent variable we can achieve that $a=0, b=r>0$, and by a linear transformation of $f(z)$ we can achieve that $f(0)=f(r)=0$. We introduce now the solutions $u_{1}(z)$ and $u_{2}(z)$ of the differential equation (2) and express $f(z)$ as their ratio

$$
f(z)=\frac{u_{1}(z)}{u_{2}(z)} .
$$

The non-univalence of $f(z)$ leads to the conclusion

$$
u_{1}(0)=u_{1}(r)=0 .
$$

To utilize this equation we map the unit disk onto the strip

by means of the function

$$
-\frac{1}{4} \pi<\operatorname{Im}\{t\}<\frac{1}{4} \pi
$$

$$
z=g(t)=\tanh t, \quad t=\frac{1}{2} \log \frac{1+z}{1-z}
$$

The points $z=0$ and $z=r$ go into the points $t=0, t=\frac{1}{2} \log ((1+r) /(1-r))=\varrho>0$ on the real axis. By virtue of (10) we know that the functions 


$$
v_{v}(t)=u_{\nu}[g(t)] g^{\prime}(t)^{-\frac{1}{2}}
$$

are defined in the strip (13) and satisfy the differential equation

$$
v_{\nu}^{\prime \prime}(t)+\frac{1}{2} S_{t}(t) v_{v}(t)=0
$$

where $S_{t}(t)$ is obtained from $S(z)$ by the transformation (8). Equation (12) implies

$$
v_{1}(0)=v_{1}(\varrho)=0
$$

Consider equation (16) for $v=1$, multiply it by $\overline{v_{1}(t)}$ and integrate the result along the real $t$-axis from 0 to $\varrho$. Integration by parts and the boundary conditions (17) lead to

$$
\frac{1}{2} \int_{0}^{e} S_{t}(t)\left|v_{1}(t)\right|^{2} d t=\int_{0}^{e}\left|v_{1}^{\prime}(t)\right|^{2} d t \geqslant 0 .
$$

We compute now the factor $S_{t}(t)$ by means of (8) and (14). We find by an easy computation

$$
S_{t}(t)=S(z) \cdot\left(1-z^{2}\right)^{2}-2 .
$$

In particular, if on the real axis

$$
|S(z)|<\frac{2}{\left(1-z^{2}\right)^{2}}
$$

we clearly have $\operatorname{Re}\left\{S_{t}(t)\right\}<0$ for real $t$ and the inequality (18) is impossible. On the other hand, the inequality

$$
|S(z)||d z|^{2}<\frac{2|d z|^{2}}{\left(1-|z|^{2}\right)^{2}}
$$

is invariant under linear transformations in view of (1.12) and the invariance of the nonEuclidean line element in the unit disk. Thus, if the Schwarzian differential parameter $S(z)=\{f, z\}$ satisfies the inequality

$$
|\{f, z\}|<\frac{2}{\left(1-|z|^{2}\right)^{2}}, \quad|z|<1
$$

we can assert that $f(z)$ cannot take the same value at two different points in the unit disk and $f(z)$ must be univalent.

$\left(21^{\prime}\right)$ is Nehari's sufficient condition for the univalence of $f(z)$. It is also known that the inequality $[5,13]$

$$
|\{f, z\}| \leqslant \frac{6}{\left(1-|z|^{2}\right)^{2}}
$$

is necessary for univalence. However, the gap between the two conditions (21') and (22) cannot be narrowed since Hille showed that [11] 


$$
f(z)=A\left(\frac{1-z}{1+z}\right)^{\delta}, \quad\{f, z\}=\frac{2\left(1-\delta^{2}\right)}{\left(1-z^{2}\right)^{2}}
$$

is univalent for real $\delta$, but non-univalent for arbitrarily small imaginary $\delta$. Thus, the constant 2 in the sufficient condition (21') cannot be replaced by any larger one.

We gave the above derivation of $\left(21^{\prime}\right)$ to show how useful the covariance of the auxiliary functions $u_{v}(z)$ can be. It allows us a great freedom in simplifying transformations and a clear understanding of the meaning of $\left(21^{\prime}\right)$. The reader may deduce from (18) many other (though not so elegant) conditions on $\{f, z\}$ which would ensure the univalence of $f(z)$.

3. As we have shown in Section I.2, we have Schwarzian conections on every Riemann surface $\mathfrak{R}$. We may thus consider the second order differential equation

$$
\frac{d^{2} u_{z}}{d z^{2}}+\frac{1}{2} S_{z}(z) u_{z}(z)=0
$$

in each neighborhood with a uniformizing parameter $z$ and continue the differential equation into adjacent neighborhoods with $z=g(t)$ through the transformation laws

$$
\left.\begin{array}{rl}
u_{t}(t) & =u_{z}[g(t)]\left[g^{\prime}(t)\right]^{-\frac{1}{2}}, \\
S_{t}(t) d t^{2} & =S_{z}(z) d z^{2}+\{z, t\} d t^{2} .
\end{array}\right\}
$$

Thus we can express the differential equation (24) in an invariant manner as

$$
\frac{d^{2} u(p)}{d p^{2}}+\frac{1}{2} S(p) u(p)=0
$$

for all points $p \in \mathfrak{R}$.

We start with an arbitrary but fixed pair $u_{\nu}(p), \nu=1,2$, of independent solutions of $\left(24^{\prime}\right)$ and continue them analytically along a closed path $\Gamma$ on the surface. By the principle of permanence of analytic relations the functions will remain solutions of (24') under this continuation, and on returning to the starting point on $\Gamma$ we will arrive with new solution functions $u_{v}^{(\Gamma)}(p)$. However, these new determinations must be linear combinations of the original solution set $u_{v}(p)$. Thus we have

$$
u_{\nu}^{(\Gamma)}(p)=A_{\nu 1}(\Gamma) u_{1}(p)+A_{v 2}(\Gamma) u_{2}(p)
$$

The matrix

$$
A(\Gamma)=\left(\begin{array}{ll}
A_{11}(\Gamma) & A_{12}(\Gamma) \\
A_{21}(\Gamma) & A_{22}(\Gamma)
\end{array}\right)
$$

is complex-valued and depends on the cycle $\Gamma$ described. 
We consider the Wronskian

$$
W(p)=u_{1}(p) u_{2}^{\prime}(p)-u_{2}(p) u_{1}^{\prime}(p) .
$$

It is easily seen that in view of the law of transformation (25) this expression is independent of the choice of uniformizers and that because of the differential equation (24) it is a constant. We may assume without loss of generality that $W(p) \equiv 1$. It then follows from (26) and (27) that

$$
W^{(\Gamma)}(p)=\|A(\Gamma)\| \cdot W(p), \quad\|A(\Gamma)\|=1 .
$$

The transformation matrices for the fundamental system $u_{\nu}(p)$ are unimodular for every cycle $\Gamma$. Thus there exists one constraint among the four elements of the matrix $A(\Gamma)$, and we find that each such matrix depends on three independent complex parameters.

If $\mathfrak{R}$ is of genus $g$, we can select a canonical set of cross cuts $\left\{\mathfrak{A}_{p}, \mathfrak{B}_{\nu}\right\}, \nu=1, \ldots, g$, and express each homotopy class of curves on $\Re$ in terms of the homotopy classes of these cross cuts, i.e., we take the homotopy classes of the cross cuts as generators of the funda-

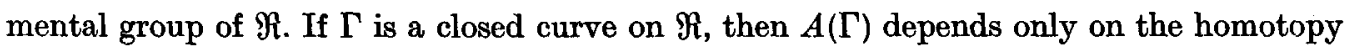
class of $\Gamma$ and not on $\Gamma$ itself. It is therefore sufficient to study the $2 g$ unimodular matrices $A\left(\mathfrak{H}_{y}\right)$ and $A\left(\mathfrak{B}_{\nu}\right)$. We note that the transformation matrices of different cross cuts do not necessarily commute, and the matrix $A(\Gamma)$ of curve $\Gamma$ depends on the homotopy class of $\Gamma$ and not merely on the homology class of the cycle which $\Gamma$ gives rise to. The study of the various matrices $A(\Gamma)$ leads therefore to a deeper theory of the moduli of a Riemann surface than that of the period matrices of Abelian integrals.

The $2 g$ matrices $A\left(\mathfrak{A}_{\nu}\right), A\left(\mathfrak{B}_{\nu}\right)$ depend on $6 g$ complex parameters. However, we have a certain freedom in the choice of the solutions $u_{\nu}(p)$ whose transformations they represent. A change of the fundamental system $u_{\nu}(p)$ leads to a similarity transformation

$$
\hat{A}(\Gamma)=M^{-1} A(\Gamma) M
$$

of the corresponding matrices. It is easily seen that $M$ contains three essential complex parameters such that the $2 g$ matrices depend on $6 g-3$ complex numbers.

We may count, on the other hand, the freedom in the choice of Schwarzian connections. Let $S(p)$ be the specific connection constructed in (1.19) from the Abelian integral of the third kind. Then the most general Schwarzian connection which is regular on $\mathfrak{R}$ is of the form

$$
S\left(p ; \lambda_{v}\right)=S(p)+\sum_{\nu=1}^{3 g-3} \lambda_{v} Q_{v}(p)
$$

where the $Q_{\nu}(p)$ are a base for all quadratic differentials, regular on $\mathfrak{R}$. We see that the Schwarzian connections form a linear manifold depending on $3 g-3$ complex parameters. 
It would be more precise to denote the matrices $A$ as $A\left(\Gamma ; \lambda_{p}\right)$ to describe the cycle as well as the specific differential equation from which they arise. Finally, it should be observed that the transformation matrices $A\left(\Gamma ; \lambda_{\nu}\right)$ depend also on the moduli of the Riemann surface $\Re$ considered. The number of these moduli is well known to be $3 g-3$. Thus the $6 g-3$ complex parameters which determine the transformation matrices depend on the following $6 g-6$ complex parameters: The $3 g-3$ moduli of the surface and the $3 g-3$ accessory parameters $\lambda_{\nu}$.

One would therefore expect that three further relations should hold between the parameters determining the $2 g$ matrices $A\left(\Gamma ; \lambda_{\nu}\right)$. This is indeed the case since the totality of cross cuts transforms $\mathfrak{R}$ into a simply-connected domain and the continuation of each $u_{\nu}(p)$ along the boundary of this domain must return each function to its initial value. Thus, under proper numeration of the cross cuts, we must have the relation

$$
\prod_{\alpha=1}^{\mathfrak{R}} A\left(\mathfrak{U}_{\alpha} ; \lambda_{\nu}\right) A\left(\mathfrak{B}_{\alpha} ; \lambda_{\nu}\right) A^{-1}\left(\mathfrak{A}_{\alpha} ; \lambda_{\nu}\right) A^{-1}\left(\mathfrak{B}_{\alpha} ; \lambda_{\nu}\right)=I
$$

which represents the sought additional three constraints on the elements of the unimodular matrices.

The enumeration of parameters at our disposal and of the essential parameters in the transformation matrix set shows that the set $A\left(\mathfrak{A}_{\nu} ; \lambda_{\mu}\right), A\left(\mathfrak{B}_{\nu} ; \lambda_{\mu}\right)$ may be considered as a possible set of moduli for the surface $\Re$. On the other hand, we are led to the interesting problem of determining those coefficient vectors $\lambda_{\nu}$ which lead to important classes of transformation groups. For example, the question arises how to determine those $\lambda_{\nu}$ whose corresponding solution set $u_{\nu}(p)$ has as ratio the polymorphic function $\varphi(p)$, discussed in Section I.2, which maps the universal covering surface of $\Re$ onto the unit disk.

4. Let us consider a domain $D$ in the complex plane and let $g(z, \zeta)$ be its Green's function. We form the analytic kernel $[5,8,20]$

$$
L(z, \zeta)=-\frac{2}{\pi} \frac{\partial^{2} g(z, \zeta)}{\partial z \partial \zeta}=\frac{1}{\pi(z-\zeta)^{2}}-l(z, \zeta)
$$

which plays a central role in the theory of the Bergman kernel function. $l(z, \zeta)$ is regular analytic in $D$ and $L(z, \zeta)$ has a double pole at $z=\zeta$, as is explicitly displayed in (31).

From the conformal invariance of the Green's function follows that under a mapping $z=g(t), \zeta=g(\tau)$ holds

$$
L(z, \zeta) d z d \zeta=\hat{L}(t, \tau) d t d \tau
$$

In terms of $l(z, \zeta)$ this leads to the transformation law 


$$
l(z, \zeta) d z d \zeta=l(t, \tau) d t d \tau+\frac{1}{\pi}[z, \zeta ; t, \tau] d t d \tau
$$

where $[z, \zeta ; t, \tau]$ is defined in (1.6). In particular, by $\left(1.8^{\prime}\right)$ we have

$$
l(z, z) d z^{2}=l(t, t) d t^{2}-\frac{1}{6 \pi}\{z, t\} d t^{2} .
$$

We recognize that

$$
S(z)=6 \pi l(z, z)
$$

transforms as a Schwarzian connection.

Let us suppose that the boundary of the domain is analytic and admits a parametrization $z=z(s)$. Denoting

$$
\dot{z}=\frac{d z}{d s}
$$

we can easily derive from the boundary behavior of the Green's function that

$$
\begin{array}{r}
L(z, \zeta) \dot{z} \dot{\zeta}=\text { real, } \quad z, \zeta \in \partial D, z \neq \zeta . \\
\text { Hence, } \quad \operatorname{Im}\{l(z, \zeta) \dot{z} \zeta\}=\frac{1}{\pi} \operatorname{Im}\left\{\frac{\dot{z} \dot{\zeta}}{(z-\zeta)^{2}}\right\} .
\end{array}
$$

The right-hand side depends here in an elementary way on the geometry of $\partial D$, while the left-hand term is an expression involving the much deeper Green's function of the domain. In particular, letting $z=\zeta$ and making an elementary computation, we find

$$
\operatorname{Im}\left\{l(z, z) \dot{z}^{2}\right\}=\frac{1}{6 \pi} \frac{d}{d s} x(s), \quad z=z(s)
$$

where $x(s)$ is the curvature of the boundary curve at $z(s)$.

We are now able to understand the significance of the differential equation

$$
u^{\prime \prime}(z)+\left[3 \pi l(z, z)+\sum \lambda_{\nu} Q_{\nu}(z)\right] u(z)=0
$$

with real $\lambda_{\nu}$ and where the $Q_{\nu}(z)$ are a basis for all real quadratic differentials of $D$, i.e., of all $Q(z)$ which satisfy on $\partial D$ the condition

$$
Q(z) \dot{z}^{2}=\text { real }
$$

Indeed, let $C_{a}$ be a component curve of $\partial D$. We may assume without loss of generality that $C_{\alpha}$ is the real axis since this can always be achieved by a conformal mapping and 
since the covariance of the solutions $u(z)$ of (40) is given by (25). By virtue of (39) and (41) we see that $u(z)$ satisfies on the real axis a second-order differential equation with real coefficients. We may therefore choose a basic solution set $u_{\nu}(z)$ which is real on the real axis and find as the general solution

$$
u(z)=A u_{1}(z)+B u_{2}(z)
$$

with arbitrary complex constants $A$ and $B$. The ratio of two independent solutions of (40) is by (25) a conformal invariant. If we denote

$$
\frac{u_{1}(z)}{u_{2}(z)}=r_{\alpha}(z)=\text { real } \text { for } z \in C_{\alpha}
$$

we find for any two solutions $u(z)$ and $v(z)$ of $(40)$ the ratio

$$
R(z)=\frac{u(z)}{v(z)}=\frac{A^{(\alpha)} r_{\alpha}(z)+B^{(\alpha)}}{C^{(\alpha)} r_{\alpha}(z)+D^{(\alpha)}} \quad \text { on } C_{\alpha} .
$$

The values of the ratio $R(z)$ on $C_{\alpha}$ lie therefore always on a circle.

There are various canonical mappings of a domain $D$ which transform boundary curves into circles. All of them may be obtained by solving the second-order differential equation (40). We are led again to the problem of determining those $\lambda_{v}$ which lead to a univalent mapping of $D$ on the canonical circular domain. The significance of differentials of order $-\frac{1}{2}$ in the theory of such canonical mappings is evident.

5. It should be pointed out that the concept of differentials of half-integer order is implicit in the general theory of the Schwarzian derivative. Indeed, let us consider the third-order nonlinear differential equation

$$
\{w, z\}=S(z)
$$

for given $S(z)$ and unknown $w=f(z)$. If $f(z)$ solves (45), one defines

$$
u_{1}(z)=f^{\prime}(z)^{-\frac{z}{2}}, \quad u_{2}(z)=f(z) f^{\prime}(z)^{-\frac{1}{2}}
$$

and proves that both functions $u_{\nu}(z)$ satisfy the same linear second-order differential equation $[2$, p. 311]

$$
u^{\prime \prime}(z)+\frac{1}{2} S(z) u(z)=0
$$

Likewise our considerations regarding the group of linear transformations of the $u_{\nu}(z)$ under homotopy classes of paths of the Riemann surface $\Re$ considered are closely related to the approach of Poincaré and Klein in the study of the uniformization problem. The 
coefficients $\lambda_{v}$ in the Schwarzian connection (30) are the well-known accessory parameters in this theory. Instead of dealing with the linear differential equation (24), one considers usually the equivalent nonlinear differential equation of the third order for the uniformizing function $f(p)$. The parameters $\lambda_{\nu}$ in the Schwarzian connection must then be adjusted in such a way that the linear transformations of the uniformizing function under the various cycles form a Fuchsian group.

It is of interest to show that this requirement simplifies if we use the half-order differentials $u_{\nu}(p)$. Indeed, the condition on the linear transformations is that they preserve the unit circle, and this engenders the requirement that the non-Euclidean line element

$$
\frac{|d w|}{1-|w|^{2}}=d s
$$

be unchanged if $w=f(p)$ undergoes its linear transformations for any closed trajectory. Since by (4) we may express $w(p)$ as the ratio of the $u_{\nu}(p)$, we find

$$
d s=\frac{|d p|}{\left|u_{2}(p)\right|^{2}-\left|u_{1}(p)\right|^{2}}
$$

if we assume the system of solutions normalized by the condition that its Wronskian have the value 1 . Thus, the group of linear transformations must have the invariant

$$
Q\left(u_{1}, u_{2}\right)=\left|u_{1}\right|^{2}-\left|u_{2}\right|^{2}
$$

The use of the $u_{\nu}(p)$ leads to a very short proof of a theorem of Poincarés which we may paraphrase: The groups of linear transformations engendered by the solution vectors $u_{\nu}(p)$ and $v_{\nu}(p)$ of the differential equations

$$
u^{\prime \prime}+\frac{1}{2} S u=0, \quad v^{\prime \prime}+\frac{1}{2} T v=0
$$

coincide only if the Schwarzian connections $S$ and $T$ are identical.

Indeed, suppose that for given Schwarzian connections $S$ and $T$ we have solution systems $u_{\nu}(p)$ and $v_{\nu}(p)$ with the same unimodular transformation matrices $A\left(\mathfrak{U}_{\alpha}\right), A\left(\mathfrak{B}_{\alpha}\right)$. We form the determinant

$$
\delta(p)=u_{1} v_{2}-u_{2} v_{1}
$$

which is a reciprocal differential on $\Re$. Under any cycle $\Gamma$ on $\Re$ we find $\delta(p)$ unchanged since the vectors $u_{\nu}(p)$ and $v_{\nu}(p)$ transform cogrediently and since the determinant of each linear transformation is by construction exactly 1 . Hence we have constructed a regular and single-valued reciprocal differential on $\mathfrak{H}$. This is clearly impossible by the Riemann- 
Roch theorem if the genus $g$ is $>1$. Thus, necessarily $\delta(p) \equiv 0$, the vectors $u_{\nu}(p)$ and $v_{\nu}(p)$ are linearly dependent and the Schwarzian derivatives of their ratios are equal. This proves $S \equiv T$ as asserted.

A proof can also be given for the case $g=1$. We omit this step since our main purpose was to show the usefulness of the $u_{v}(p)$. Again we have only paraphrased a standard proof of the Poincaré theorem [2], but a comparison of the arguments will show how the explicit use of half-order differentials has been illuminating.

6. The limitation of our consideration to second-order equations of the form of (24) or, as invariantly expressed, $\left(24^{\prime}\right)$-may seem at first to be an arbitrary restriction. This is not the case, as we shall show. In fact any second-order, linear, homogeneous, differential equation which is invariantly defined and everywhere regular on a compact Riemann surface must be of the form

$$
\frac{d^{2} u}{d p^{2}}+\frac{1}{2} S(p) u=0
$$

where $S(p)$ transforms as

$$
S_{t}=S_{z}\left(\frac{d z}{d t}\right)^{2}+\{z, t\}
$$

here, as before, $S_{t}$ is the representation of $S(p)$ in terms of the local uniformizer $t$, and $S_{z}$ is the representation of $S(p)$ in terms of the local uniformizer $z$.

To be more specific, consider a general second-order, linear, homogeneous, differential equation on the Riemann surface. Let its representation in terms of $t$ be

$$
v^{\prime \prime}+p v^{\prime}+q v=0
$$

and its representation in terms of $z$ be

$$
u^{\prime \prime}+P u^{\prime}+Q u=0
$$

where ' denotes differentiation with respect to the obvious argument. We require that the coefficients in the differential equation transform according to a linear inhomogeneous law under change of uniformizer

$$
p=\alpha P+\beta, \quad q=\gamma Q+\delta
$$

with coefficients which depend on the relation between $z$ and $t$ only. The dependent variable shall transform according to a linear homogeneous rule

$$
v(t)=\Phi(t) u[z(t)]
$$

Thus

$$
v^{\prime}=\Phi u^{\prime} z^{\prime}+\Phi^{\prime} u
$$

14-662945 Acta mathematica. 115. Imprimé le 11 mars 1966. 
which means

$$
\begin{gathered}
\frac{d v(t)}{d t}=\Phi(t) \frac{d u(z)}{d z} \frac{d z}{d t}+\Phi^{\prime}(t) u(z) \\
v^{\prime \prime}=\Phi z^{\prime 2} u^{\prime \prime}+\left(2 \Phi^{\prime} z^{\prime}+\Phi z^{\prime \prime}\right) u^{\prime}+\Phi^{\prime \prime} u
\end{gathered}
$$

We also have

Upon substituting from (58) and (59) into (55) we get

$$
\Phi z^{\prime 2} u^{n}+\left(2 \Phi^{\prime} z^{\prime}+\Phi z^{n}+p \Phi z^{\prime}\right) u^{\prime}+\left(\Phi^{n}+\Phi^{\prime} p+\Phi q\right) u=0 .
$$

Since the differential equation is invariant, we can immediately conclude, upon comparing (56) and (60), that

and

$$
\begin{gathered}
\Phi^{\prime \prime}+\Phi^{\prime} p+\Phi q=\Phi z^{\prime 2} Q \\
2 \Phi^{\prime} z^{\prime}+\Phi z^{\prime \prime}+p \Phi z^{\prime}=\Phi z^{\prime 2} P
\end{gathered}
$$

Since $Q(z)$ depends on $t$ and $q$, but certainly not on $p$, we may conclude from (61) that either

(i) $\Phi \equiv$ constant $\quad$ (so $\Phi^{\prime} \equiv 0$ )

or (ii) $p \equiv 0$.

Let us examine the consequences of (i) first. In this case (61) reduces to

$$
q(t)=Q(z) z^{\prime 2}
$$

thus $q$, i.e., the last coefficient in the differential equation, is a quadratic differential. Also, equation $(62)$ becomes

$$
p(t)=P(z) z^{\prime}-\frac{z^{\prime \prime}}{z^{\prime}}
$$

This means that $p$, i.e., the coefficient of the first order term, is a connection, see [18, p. 251].

But it is known (see [18, p. 252]) that the sum of the residues of a connection on a compact Riemann surface of genus $g$ is $2-2 g$; therefore, if $g \neq 1$ the connection must have singularities. Thus the differential equation (55) must be singular unless either $g=1$, the case of elliptic function theory, or $p \equiv 0$, which brings us to case (ii). Before we consider case (ii), let us mention that since $\Phi \equiv$ const, we can take $\Phi \equiv 1$, which means that the solutions of (55) are functions, at least locally, i.e., they transform like functions.

In considering case (ii) we again return to equation (61) which now becomes

And equation (62) becomes

$$
\frac{\Phi^{\prime \prime}}{\Phi}+q=Q z^{\prime 2}
$$

$$
2 \Phi^{\prime} z^{\prime}+\Phi z^{\prime \prime}=0
$$


This means that

$$
\Phi=k z^{\prime-\frac{1}{2}}
$$

where $k$ is a constant which we choose to be 1 . In this case an easy calculation shows that

$$
\frac{\Phi^{\prime \prime}}{\Phi}=-\frac{1}{2}\{z, t\}
$$

so that ( 61 ii) becomes

$$
q=Q \cdot z^{2}+\frac{1}{2}\{z, t\}
$$

and setting

$$
q=\frac{1}{2} S_{t} \quad \text { and } \quad Q=\frac{1}{2} S_{z}
$$

we have established our assertion. In case the equation (55) is regular, i.e., $p \equiv 0$ (in all coordinates), then $\Phi=\left(z^{\prime}\right)^{-\frac{1}{2}}$, so the solutions of (55) transform like reciprocal half-order differentials, i.e., differentials of order $-\frac{1}{2}$.

Finally, we should mention that the case $g=1$ has not been overlooked (in fact, we consider it a very important testing ground for general theories-as it has been for more than a century!). Although one nonsingular connection does exist in this case, we may use a uniformizer on the surface which makes it equal to zero everywhere, which brings us back to the case (ii) again. All other connections in the case $g=1$ are singular, so our assertion is established with complete generality.

7. In this section we wish to make precise the concept of analytic continuation of differentials of order $\pm \frac{1}{2}$ over the Riemann surface. This consideration is necessary in order to define clearly the meaning of the transformation matrices $A(\Gamma)$ introduced in Section 3 of this chapter.

If we consider the Riemann surface $\mathfrak{H}$ realized as a covering over the complex $z$-plane, we might define the half-order differentials locally as analytic functions of $z$ and their continuation over $\mathfrak{R}$ as explicit analytic continuation. However, for the general theory of abstract Riemann surfaces the following argument may be more appropriate.

In order to save space, we adopt the notations and definitions given in [18, pp. 249-51]. Thus, by a differential of order $\frac{1}{2}$ we mean a collection of variables $\left\{\psi_{\alpha}\right\}$ which satisfy the transformation laws

$$
\psi_{\alpha}=\psi_{\beta}\left(\frac{d z_{\beta}}{d z_{\alpha}}\right)^{\frac{1}{2}} \text { in } U_{\alpha} \cap U_{\beta}
$$

By $\left(d z_{\beta} / d z_{\alpha}\right)^{\frac{1}{2}}$ we mean an analytic function in $U_{\alpha} \cap U_{\beta}$ whose square is $d z_{\beta} / d z_{\alpha}$. Of course, this function is not unique (there are two choices in each case) and we must show that we can choose them consistently, i.e., such that

$$
\left(\frac{d z_{\beta}}{d z_{\alpha}}\right)^{\frac{1}{2}}\left(\frac{d z_{\gamma}}{d z_{\beta}}\right)^{\frac{1}{2}}\left(\frac{d z_{\alpha}}{d z_{\gamma}}\right)^{\frac{1}{2}} \equiv 1 \quad \text { in } U_{\alpha} \cap U_{\beta} \cap U_{\gamma}
$$


In order to show that we can make such a choice, let $\theta_{\alpha \beta}$ be any collection of analytic functions chosen on the sets $U_{\alpha} \cap U_{\beta}$ such that

$$
\theta_{\alpha \beta}^{2}=\frac{d z_{\beta}}{d z_{\alpha}} \text { in } U_{\alpha} \cap U_{\beta}
$$

Then

$$
\theta_{\alpha \beta} \theta_{\beta \gamma} \theta_{\gamma \alpha} \equiv s_{\alpha \beta \gamma}= \pm 1 \text { for } U_{\alpha} \cap U_{\beta} \cap U_{\gamma} \neq \varnothing .
$$

Let $N(\mathfrak{U})$ be the nerve of covering $\mathfrak{U}=\left\{U_{\alpha}\right\}$, and $\mathfrak{U}$ chosen as in [18, p. 255]. Then $s_{\alpha \beta \gamma}$ determines a 2 -cocycle on $N(\mathfrak{U})$ given by

$$
s\left[\sigma_{\alpha \beta \gamma}\right]=s_{\alpha \beta \gamma}
$$

which in turn determines an element of $H^{2}(\Re, G)$, the two-dimensional cohomology group of $\mathfrak{R}$ with coefficients in $G$, the multiplicative group consisting of the two elements 1 and -1 .

Clearly, we have

$$
\prod_{\sigma^{2}} s\left[\sigma^{2}\right]=1
$$

where the product is taken over all the positively oriented $\sigma^{2}$ in $N(\mathfrak{U})$. Indeed $\prod_{\sigma} s[\sigma]$ as given in (68) represents the product of all the $s_{\alpha \beta \gamma}$. But each $\sigma_{\alpha \beta}$ occurs as the face of exactly two two-simplexes, say $\sigma_{\alpha \beta \gamma}$ and $\sigma_{\beta \alpha \delta}$ (see illustration [18, p. 255]). Since $\theta_{\alpha \beta}$ and $\theta_{\beta \alpha}=1 / \theta_{\alpha \beta}$ each occurs once in the product, equation (68) holds. But this equation means that $s$ is cohomologous to the identity (upon using the fact that $\mathfrak{R}$ is an orientable, twodimensional manifold), i.e.,

$$
s_{\alpha \beta \gamma}=s_{\alpha \beta} s_{\beta \gamma} s_{\gamma \alpha}, \quad U_{\alpha} \cap U_{\beta} \cap U_{\gamma} \neq \varnothing,
$$

where $s_{\alpha \beta}= \pm 1$, etc.

$$
\text { Now define } \quad\left(\frac{d z_{\beta}}{d z_{\alpha}}\right)^{\frac{1}{2}}=s_{\alpha \beta} \theta_{\alpha \beta},
$$

then clearly

$$
\left(\frac{d z_{\beta}}{d z_{\alpha}}\right)^{\frac{1}{2}}\left(\frac{d z_{\gamma}}{d z_{\beta}}\right)^{\frac{1}{2}}\left(\frac{d z_{\alpha}}{d z_{\gamma}}\right)^{\frac{1}{2}} \equiv 1 \quad \text { in } U_{\alpha} \cap U_{\beta} \cap U_{\gamma}
$$

since

$$
s_{\alpha \beta} \theta_{\alpha \beta} s_{\beta \gamma} \theta_{\beta \gamma} s_{\gamma \alpha} \theta_{\gamma \alpha} \equiv s_{\alpha \beta \gamma} s_{\alpha \beta \gamma}=1 \text {. }
$$

We have thus defined a coherent set of expressions $\left(d z_{\beta} / d z_{\alpha}\right)^{\frac{1}{2}}$. By defining

$$
u_{\alpha}=u_{\beta}\left(\frac{d z_{\beta}}{d z_{\alpha}}\right)^{\frac{1}{2}}
$$

we can now give an unambiguous definition to differentials of order $\frac{1}{2}$ on $\mathfrak{R}$.

The differentials of order $-\frac{1}{2}$ can be defined by the same procedure. 


\section{The Szegö kernel of a Riemann surface}

1. Let $D$ be a planar domain bounded by a finite set of analytic curves. In the boundary value problems for analytic functions in $D$, as well as in the theory of conformal mapping, one can very successfully apply the theory of orthogonal analytic functions and their kernels. The most important norms used in such theories are those involving area integrals

$$
(\varphi, \psi)=\iint_{D} \varphi(z) \overline{\psi(z)} d x d y, \quad z=x+i y
$$

and boundary line integrals

$$
((\varphi, \psi))=\int_{\partial D} \varphi(z) \overline{\psi(z)} d s
$$

If we have a complete orthonormal set of analytic functions $\varphi_{\nu}(z)$ in $D$, we can form their kernel $[3,4,8,20]$

$$
K(z, \bar{\zeta})=\sum_{\nu=1}^{\infty} \varphi_{\nu}(z) \overline{\varphi_{\nu}(\zeta)}
$$

which converges in both cases almost uniformly in $D$ and is independent of the particular choice of the complete orthonormal set. For normalization (1) one obtains the Bergman kernel which is closely related to the Green's function of $D$ and which has many applications in the theory of conformal mapping. In the case of normalization (2) one obtains a kernel which was first introduced by Szegö [21]. Garabedian [9] showed the close connection between the Bergman and the Szegö kernels in the case of planar domains.

We can characterize the Bergman kernel by the reproducing property

$$
\iint_{D} K_{B}(z, \bar{\zeta}) f(\zeta) d x d y=f(z)
$$

and the Szegö kernel by the analogous equation

$$
\int_{\partial D} K_{S}(z, \zeta) f(\zeta) d s=f(z)
$$

In order to study the behavior of the kernels under conformal mapping and with the aim of extending the theory to domains on Riemann surfaces we shall write (4) and (5) in the form

$$
\iint_{D} K_{B}(z, \bar{\zeta}) f^{\prime}(\zeta) d x d y=f^{\prime}(z)
$$

and

$$
\int_{\partial D} K_{S}(z, \bar{\zeta}) \sqrt{f^{\prime}(\zeta)} d s=\sqrt{f^{\prime}(z)}
$$


where we assume that $\sqrt{f^{\prime}(z)}$ is a single-valued analytic function in $D$. If we now define the transformation laws

$$
\begin{gathered}
\hat{K}_{B}(w, \bar{\omega}) d w d \bar{\omega}=K_{B}(z, \bar{\zeta}) d z d \bar{\zeta} \\
\hat{K}_{S}(w, \bar{\omega}) d w^{\frac{1}{z}} d \bar{\omega}^{\frac{1}{t}}=K_{S}(z, \bar{\zeta}) d z^{\frac{1}{4}} d \bar{\zeta}^{\frac{1}{2}}
\end{gathered}
$$

it can easily be seen that the reproducing properties $\left(4^{\prime}\right)$ and $\left(5^{\prime}\right)$ are preserved under conformal mapping. We see, in particular, that a more penetrating theory of the Szegö kernel leads necessarily to the consideration of differentials of half-integer order.

2. It is well known that the function theory of planar domains can be easier understood if we complete them to compact Riemann surfaces by adding to them their double. In particular, the Green's function and the Bergman kernel can be expressed in terms of certain Abelian integrals and differentials of the symmetric Riemann surface so obtained. We therefore shall start with an arbitrary closed Riemann surface $\Re$ and consider there such expressions which for the case of symmetric surfaces will reduce to differentials like the Bergman and the Szegö kernel.

We start with the symmetric Abelian integral $W(p, r ; q, s)$ of the surface $\mathfrak{R}$, as we defined in Section I.2, and form the double differential

$$
L(p, q)=\frac{\partial^{2} W(p, r ; q, s)}{\partial p \partial q}
$$

which is independent of $r, s$ and symmetric in $p, q$. It is regular for $p, q \in \Re$, except for the case $p=q$ when we have a double pole as indicated in (1.17). This double differential is closely related to the Bergman kernel in the case of planar domains; we may therefore refer to it as the Bergman kernel of $\mathfrak{R}$.

We wish now to construct correspondingly a Szegö kernel for the Riemann surface which shall be a half-order differential in each variable, have a simple pole if both arguments coincide and which is anti-symmetric in $p$ and $q$. Let us denote it by $\Lambda(p, q)$. Clearly, $\Lambda(p, q)^{2}$ will be a double differential on $\Re$ with all the regularity and symmetry properties of the Bergman kernel $L(p, q)$. If it exists at all, it must have the form

$$
\Lambda(p, q)^{2}=L(p, q)+\sum_{i, k=1}^{g} a_{i k} w_{i}^{\prime}(p) w_{k}^{\prime}(q),
$$

where the $w_{f}^{\prime}(p)$ are the normalized Abelian differentials of the first kind of $\mathfrak{R}$ and where the $a_{i k}$ form a symmetric matrix. Since $L(p, q)$ and the Abelian differentials $w_{i}^{\prime}(p)$ are well studied, we are led to the algebraic problem to form a combination of $L(p, q)$ and the $g$ differential $w_{i}^{\prime}(p)$, which has only double zeros on $\Re$. 
An algebraic approach to this problem is as follows. Let $p_{k}(k=1,2, \ldots, g)$ be a set of points on $\Re$ and form the determinant

$$
D\left(p ; q ; p_{j}\right)=\left\|\begin{array}{ll}
L(p, q) & w_{i}^{\prime}(p) \\
L\left(p_{k} ; q\right) & w_{i}^{\prime}\left(p_{k}\right)
\end{array}\right\|
$$

of $g+1$ rows and $g+1$ columns. This expression represents for fixed $q$ and $p_{k}$ a differential of the first order on $\mathfrak{R}$ with the $g$ zeros $p_{k}$ and the double pole at $q$. Since a differential of the first order with a double pole has precisely $2 g$ zeros, we see that $D\left(p ; q, p_{j}\right)$ has another set of $g$ zeros on $\Re$. But we have still the freedom in the choice of the $p_{j}$ to achieve that each zero $p_{j}$ is a double zero. For this purpose, we must fulfill the $g$ conditions

$$
D^{\prime}\left(p_{\varrho} ; q, p_{j}\right)=\left\|\begin{array}{cc}
L^{\prime}\left(p_{\varrho} ; q\right) & w_{i}^{\prime \prime}\left(p_{\varrho}\right) \\
L\left(p_{k} ; q\right) & w_{i}^{\prime}\left(p_{k}\right)
\end{array}\right\|=0, \quad \varrho=1, \ldots, g
$$

We thus have $g$ equations for the $g$ zeros $p_{j}$, which shows that the problem is hopeful.

But in order to avoid the theory of elimination for algebraic functions, we shall construct $\Lambda(p, q)$ through the deeper theory of Abelian integrals and by use of the classical results on the Jacobi inversion problem. We follow here an analogous approach as was used by Garabedian in constructing the Szegö kernel of plane domains. We use the Abelian integral of the third kind $w(p ; r, s)$ defined in Section I.2. Since it is normalized to have the periods zero around each cross cut $\mathfrak{A}_{\nu}$ of an arbitrary but fixed system of canonical cross cuts, we know that it has the following periods along a cross cut $\mathfrak{B}_{\nu}$ :

$$
\int_{\mathfrak{B}_{\nu}} d w(p ; r s)=2 \pi i\left[w_{\nu}(r)-w_{\nu}(s)\right]
$$

The integrals of the first kind $w_{\nu}(p)$ are normalized with respect to the same canonical cut system such that

$$
\int_{\mathfrak{A}_{\mu}} d w_{\nu}=\delta_{\nu \mu}
$$

We select an arbitrary but fixed differential of the first kind $v^{\prime}(p)$ on $\Re$ and denote its zeros by $p_{1}, p_{2}, \ldots, p_{\rho} ; \varrho=2 g-2$. We pick another set of $g$ points $q_{1}, \ldots, q_{g}$ on $\mathfrak{R}$ and form the expression

$$
\begin{aligned}
E\left(p ; q, q_{\nu}\right)=\log v^{\prime}(p) & +\sum_{\nu=1}^{g-1}\left[w\left(p ; q_{\nu}, p_{2 \nu-1}\right)+w\left(p ; q_{\nu}, p_{2 \nu}\right)\right] \\
& +2 w\left(p ; q_{g}, q\right)+4 \pi i \sum_{\alpha=1}^{g} a_{\alpha} w_{\alpha}(p)
\end{aligned}
$$


On the right-hand side we have cancelled out all logarithmic poles at the zeros $p_{\nu}(\nu=1, \ldots$, $2 g-2)$ of the given differential $v^{\prime}(p)$. We have, however, logarithmic poles at the points $q_{\nu}(\nu=1, \ldots, g)$ with the residue +2 and a logarithmic pole at $q$ with the residue -2 .

The expression $E\left(p ; q, q_{v}\right)$ is not single valued on $\Re$. If we describe a cycle $\mathfrak{A}_{\beta}$, the $w\left(p ; q_{\nu}, p_{\mu}\right)$ do not change, but $\log v^{\prime}(p)$ may change by $2 \pi i m_{\beta}\left(m_{\beta}=\right.$ integer $)$ and the last sum increases by $4 \pi i a_{\beta}$. Under a cycle $\mathfrak{B}_{\beta}$ we have in view of (12) the period

$$
\begin{aligned}
\int_{\mathfrak{B}_{\beta}} d E\left(p ; q, q_{\nu}\right)=2 \pi i\left\{n_{\beta}\right. & +\sum_{\nu=1}^{g-1}\left[2 w_{\beta}\left(q_{\nu}\right)-w_{\beta}\left(p_{2 v-1}\right)-w_{\beta}\left(p_{2 v}\right)\right] \\
& \left.+2\left(w_{\beta}\left(q_{o}\right)-w_{\beta}(q)\right)+2 \sum_{\alpha=1}^{g} a_{\alpha} P_{\alpha \beta}\right\}
\end{aligned}
$$

if we introduce the Riemann matrix of periods for the normalized Abelian integrals of the first kind,

$$
P_{\alpha \beta}=\int_{\mathfrak{B}_{\beta}} d w_{\alpha}
$$

$n_{\beta}$ is again an integer.

We apply now the existence theorem for the Jacobi inversion problem. Given any set of $g$ complex numbers $\xi_{\beta}$, we can always find $g$ points $q_{\nu}$ on $\mathfrak{R}$ such that

$$
\sum_{\nu=1}^{g} w_{\beta}\left(q_{\nu}\right)=\xi_{\beta}+k_{\beta}+\sum_{\alpha=1}^{g} l_{\alpha} P_{\alpha \beta}, \quad \beta=1,2, \ldots, g
$$

with integers $k_{\beta}$ and $l_{\alpha}$. That is, the left-hand sum differs from the $\xi_{\beta}$ only by a period of $w_{\beta}(p)[12]$.

Given an arbitrary but fixed $q \in \mathfrak{\Re}$, we determine $q_{\nu}$ in such a way that

$$
\sum_{\nu=1}^{g} w_{\beta}\left(q_{\nu}\right)=w_{\beta}(q)+\frac{1}{2} \sum_{\rho=1}^{2 g-2} w_{\beta}\left(p_{\nu}\right)+k_{\beta}+\sum_{\alpha=1}^{g} l_{\alpha} P_{\alpha \beta}
$$

This is always possible by the inversion theorem. Finally, we determine the coefficients $a_{\alpha}$ as the integers

$$
a_{\alpha}=-l_{\alpha}
$$

With these choices of parameters we find that

$$
\int_{\mathfrak{B}_{\beta}} d E\left(p ; q, q_{\nu}\right)=2 \pi i\left(n_{\beta}+2 k_{\beta}\right)
$$

and

$$
\int_{\mathfrak{A}_{\beta}} d E\left(p ; q, q_{\nu}\right)=2 \pi i\left(m_{\beta}-2 l_{\beta}\right) .
$$


All periods of $E\left(p ; q, q_{\nu}\right)$ on $\mathfrak{R}$ are integer multiples of $2 \pi i$. Hence

$$
\lambda(p, q)=\exp \left\{E\left(p ; q, q_{\nu}\right)\right\}
$$

is single valued on $\mathfrak{R}$ and has a double pole at the chosen point $q$ and double zeros at the points $q_{\nu}$ determined by it. Since $\lambda(p, q)$ has the factor $v^{\prime}(p)$ and is else expressed in terms of Abelian integrals only, it is a differential of the first order on $\Re$ with a double pole and $g$ double zeros.

Finally, we construct the expression

$$
\Lambda(p, q)=\sqrt{\lambda(p, q)}
$$

It is a half-order differential in $p$ which is regular everywhere on $\mathfrak{R}$, except for a simple pole at $q$. It is determined only up to a \pm sign and can change its determination if we continue it over a closed cycle on $\mathfrak{R}$. It therefore will, in general, be single valued only on a proper two-sheeted covering of the surface. It is easily seen that the indeterminacy of sign comes solely from the behavior of $\sqrt{v^{\prime}(p)}$. Indeed, the change of argument of $\Lambda(p, q)$ over $\mathfrak{U}_{\beta}$ and $\mathfrak{B}_{\beta}$ is $\frac{1}{2} m_{\beta}-l_{\beta}$ and $\frac{1}{2} n_{\beta}+k_{\beta}$, respectively, as can be seen from (20) and (21). Thus, only the parity of the periods $m_{\beta}, n_{\beta}$ depending on $v^{\prime}(p)$ decides the changes of sign in $\Lambda(p, q)$.

Hence, if we construct $\Lambda(p, q)$ for different values of $q$ but with the same differential of the first kind $v^{\prime}(p)$, the two-sheeted covering of $\mathfrak{\Re}$ will always be the same.

3. We normalize the half-order differential $\Lambda(p, q)$ by the requirement that in a uniformizer neighborhood of $q$ we have

$$
\Lambda(p, q) d p^{\frac{1}{2}} d q^{\frac{1}{2}}=\frac{\sqrt{d z d \zeta}}{z-\zeta}+\text { regular differential }
$$

It is easily seen that this normalization is independent of the specific parameter used.

Let now $\Lambda(p, q)$ and $\Lambda\left(p, q_{1}\right)$ be any two half-order differentials on $\mathfrak{R}$ with simple poles at $q$ and $q_{1}$, respectively, the normalization (24) and both single valued on the same two-sheeted covering of $\Re$. In this case the product $\Lambda(p, q) \Lambda\left(p, q_{1}\right)$ is a single-valued differential on $\Re$ with two simple poles at $q$ and $q_{1}$. Hence the sum of its residues must equal to zero and we find

We thus proved

$$
\Lambda\left(q_{1}, q\right)+\Lambda\left(q, q_{1}\right)=0
$$

$$
\Lambda\left(q, q_{1}\right)=-\Lambda\left(q_{1}, q\right)
$$

The half-order differential $\Lambda(p, q)$ in $p$ is antisymmetric in both its arguments. It is therefore also a half-order differential in $q$. It is single valued in dependence on $q$ on the same 
two-sheeted covering of $\Re$. Our argument shows further that $\Lambda(p, q)$ is uniquely determined by the fact that it is a half-order differential in $p$ with a simple pole at $q$ and the normalization (24), provided that its sign changes on cycles on $\Re$ are prescribed. We shall call $\Lambda(p, q)$ the Szegö kernel of $\Re$ with respect to the two-sheeted covering considered.

We can construct interesting combinations of Szegö kernels which are single valued on $\mathfrak{\Re}$. Consider, for example, the expression

$$
U(p ; r, s)=\frac{\Lambda(p, r) \Lambda(p, s)}{\Lambda(r, s)}, \quad r \neq s
$$

It is a single-valued differential of first order in $p$ and a single-valued function in $r$ and $s$ on the surface $\mathfrak{R}$. Indeed, if any variable changes on $\Re$, the corresponding sign changes occur always in pairs in the product (26). The differential in $p$ has two simple poles at $r$ and $s$ with the residues +1 and -1 , respectively. It is thus an Abelian differential of the third kind, analogous to $w^{\prime}(p ; r, s)$ used before. The new differential has, however, a remarkable factorization. The identity (26) indicates the significance of the half-order differentials as building blocks for the classical single-valued differentials on a Riemann surface.

4. Given a Szegö kernel $\Lambda(p, q)$, which is single valued on a specified two-sheeted covering of $\mathfrak{R}$, we shall call two points $m$ and $n$ on $\Re$ associated if they satisfy the equation

$$
\Lambda(m, n)=0
$$

Because of the antisymmetry of the Szegö kernel this relation is a symmetric one. Each given point $q \in \Re$ has precisely $g$ associated points $q_{\nu}(\nu=1,2, \ldots, g)$ if we count them by their multiplicity.

The construction of $\Lambda(p, q)$ suggests a close relation between the set of associated points $q_{v}$ and the Jacobi inversion problem. This relation can be made more explicit by the following consideration. Let $u^{\prime}(p)$ and $v^{\prime}(p)$ be two differentials of the first kind on $\Re$. Then their ratio will be a function on $\mathfrak{M}$ and the integral

$$
\frac{1}{2 \pi i} \sum_{v} \int_{\mathfrak{A}_{v}+\mathfrak{B}_{v}} w_{k}(p) d\left[\log \frac{u^{\prime}(p)}{v^{\prime}(p)}\right]=I_{k}
$$

will be defined for every normalized integral of the first kind $w_{k}(p)$. The standard method of contour integration shows that $I_{k}$ is a period of $w_{k}(p)$ :

$$
I_{k}=n_{k}+\sum_{v=1}^{g} m_{v} P_{v k}, \quad n_{k}, m_{v} \text { integers. }
$$


On the other hand, let $\alpha_{\rho}$ be the set of zeros of $u^{\prime}(p)$ and $\beta_{\rho}$ the corresponding set of $v^{\prime}(p)$. Then the residue theorem yields

$$
I_{k}=\sum_{e=1}^{2 g-2} w_{k}\left(\alpha_{e}\right)-\sum_{e=1}^{2 g-2} w_{k}\left(\beta_{\varrho}\right), \quad k=1,2, \ldots, g .
$$

Thus the sum of each $w_{k}(p)$ extended over the set of zeros of any differential of the first kind is the same except for a period of $w_{k}(p)$.

Each integral of the first kind $w_{k}(p)$ is defined only up to an additive constant. We may normalize these integrals further as follows. Let $v^{\prime}(p)$ be the special integral of the first kind used in Section 2 to construct the Szegö kernel; we demand

$$
\sum_{\varrho=1}^{2 o-2} w_{k}\left(p_{\varrho}\right)=0
$$

where the sum is extended over all zeros $p_{\varrho}$ of $v^{\prime}(p)$. This implies

$$
\sum_{\varrho=1}^{2 g-2} w_{k}\left(\alpha_{\varrho}\right) \equiv 0 \quad\left(\bmod . \text { period of } w_{k}(p)\right),
$$

where $\alpha_{\rho}$ is the set of zeros of any differential of the first kind.

In view of (18) we find the following characterization of the set $q_{\nu}$ of points associated to $q$ :

$$
\sum_{\nu=1}^{g} w_{\beta}\left(q_{\nu}\right) \equiv w_{\beta}(q) \quad\left(\bmod . \text { period of } w_{\beta}(p)\right)
$$

The associated point set $q_{\nu}$ of $q$ solves a special Jacobi inversion probleml

The normalization (30) is obviously only possible if the genus of $\mathfrak{R}$ satisfies $g>1$ since for $g=1$ the differential of the first kind has no zeros. We shall consider this special case briefly in the following section.

We may use the concept of associated points to construct the following differentials; let $q_{\nu}$ be one point associated to $q$ and form

$$
v_{v}^{\prime}(p)=\Lambda(p, q) \Lambda\left(p, q_{\nu}\right)
$$

It is easily seen that $v_{y}^{\prime}(p)$ is a differential of first order in $p$, single valued on $\mathfrak{R}$ and regular everywhere. Indeed, the poles of the factors are just cancelled out because of the relation $\Lambda\left(q, q_{v}\right)=0$. Thus we can construct $g$ differentials of the first kind on $\mathfrak{R}$ by choosing $\boldsymbol{v}=1$, $2, \ldots, g$. Since $v_{\nu}^{\prime}(p)$ vanishes at all associated points $q_{\mu}$ of $q$ except for $q_{\nu}$, we see that the $g$ differentials of the first kind are linearly independent and form a basis for all differentials of the first kind. If $w^{\prime}(p)$ is a differential of the first kind, we have the development

$$
w^{\prime}(p)=\sum_{\nu=1}^{g} \frac{\Lambda\left(p, q_{\nu}\right) \Lambda(p, q)}{\Lambda^{\prime}\left(q_{\nu}, q\right)} w^{\prime}\left(q_{v}\right)
$$


4. Let us illustrate the general theory of the Szegö kernel by considering the special case of genus 1 . Here we may visualize the Riemann surface in the complex $u$-plane in the form of a parallelogram generated by the vectors $2 \omega_{1}$ and $2 \omega_{2}$ in which opposite sides are identified. We interpret the effect of a closed cycle $\mathfrak{A}$ as a parallel displacement by the vector $2 \omega_{1}$ and interpret a parallel displacement by the vector $2 \omega_{2}$ as the outcome of a $\mathfrak{B}$-cycle. We have at our disposal the elliptic functions with the periods $2 \omega_{1}, 2 \omega_{2}$ in order to construct the various Abelian integrals, and we shall use the notations of the Weierstrass theory.

We find at once that

$$
w(p)=\frac{1}{2 \omega_{1}} u
$$

is the normalized integral of the first kind and that the Riemann matrix reduces to

$$
P=\int_{0}^{2 \omega_{2}} d w=\frac{\omega_{2}}{\omega_{1}}
$$

the modulus of the parallelogram. The function

$$
t(p ; q)=\zeta(u-v)-\frac{\eta_{1}}{\omega_{1}} u, \quad v=u(q)
$$

is clearly a normalized integral of the second kind since it has a simple pole at $p=q$ and the period zero over the $\mathfrak{A}$-cycle. The expression

$$
L(p, q)=-\frac{d}{d p} t(p ; q)=\rho(u-v)+\frac{\eta_{1}}{\omega_{1}}
$$

is a differential in $p$; since it is symmetric in $p$ and $q$, it is a double differential in both variables. It has a double pole for $p=q$ and has the residue zero. We verify that

$$
\int_{\mathfrak{A}} L(p, q) d p=0
$$

from which it follows that this kernel coincides with the Bergman kernel (8) of the Riemann surface.

According to (9) and because of (34) we find for the Szegö kernel the representation

$$
\Lambda(p, q)^{2}=\wp(u-v)+\frac{\eta_{1}}{\omega_{1}}+a \cdot \frac{1}{4 \omega_{1}^{2}} .
$$

The constant $a$ has to be chosen such that the right-hand side has a double zero. Since 


$$
\wp^{\prime}(u)^{2}=4\left(\wp(u)-e_{1}\right)\left(\wp(u)-e_{2}\right)\left(\wp(u)-e_{3}\right)
$$

we may choose $a$ in three different ways:

$$
\begin{gathered}
\Lambda(p, q)^{2}=\wp(u-v)-e_{\varrho}, \quad \varrho=1,2,3 \\
a_{\varrho}=-4 \omega_{1}^{2}\left[e_{\varrho}+\frac{\eta_{1}}{\omega_{1}}\right] . \\
\sqrt{\wp(u)-e_{\varrho}}=\frac{\sigma_{\varrho}(u)}{\sigma(u)}
\end{gathered}
$$$$
\text { and }
$$

The functions

are very familiar in the theory of elliptic functions and of theta functions. Their significance is now explained by the role as the Szegö kernels of the surface. We have three different Szegö kernels for the surface $\mathfrak{R}$ since it possesses precisely three different twosheeted covering surfaces. These are obtained by assigning independently the sign +1 or -1 to the effect of a cycle $\mathfrak{A}$ or $\mathfrak{B}$ and omitting the combination $+1,+1$ which corresponds to the original Riemann surface itself.

$$
\text { Since } \quad \wp\left(\omega_{\varrho}\right)=e_{\varrho}
$$

we see that the condition for associated points $\Lambda\left(q^{*}, q\right)=0$ leads to the equation

The well-known equation

$$
v^{*}=v+\omega_{\varrho}
$$

$$
\sqrt{\wp(u)-e_{1}} \sqrt{\wp\left(u+\omega_{1}\right)-e_{1}}=-\sqrt{\left(e_{1}-e_{2}\right)\left(e_{1}-e_{3}\right)}
$$

is therefore nothing but the special case of (32) since the only differential of the first kind is a constant in our choice of uniformizer.

In the following chapter we shall discuss some relations between the Riemann period matrix $\left(\left(P_{\alpha \beta}\right)\right)$ and the matrix $\left(\left(a_{\alpha \beta}\right)\right)$ which connects the Bergman and the Szegö kernel through the identity (9). While some interesting results will be obtained, the explicit form of the coefficient $a_{\rho}$ given in $\left(41^{\prime}\right)$ shows already that the relation between the two $g \times g$ matrices is by no means elementary.

\section{The variational formula for the Szegö kernel}

1. In the preceding chapter we have shown that the Szegö kernel $\Lambda(p, q)$ is uniquely determined by the Riemann surface $\mathfrak{R}$ and the two-sheeted covering on which it is single valued. If we change $\mathfrak{R}$ continuously, we can deform simultaneously the covering in a 
corresponding fashion and are thus led to a continuous variation of the Szegö kernel. A study of this variation will then disclose the functional dependence of $\Lambda(p, q)$ upon the Riemann surface $\Re$ and its moduli.

Since there exist many ways of describing $\mathfrak{R}$ in terms of moduli, we shall use a somewhat special but very intuitive kinematics to deform $\Re$. We select an arbitrary but fixed point $r_{0} \in \Re$ and introduce at $r_{0}$ the uniformizer $z(p)$ such that $z\left(r_{0}\right)=0$. A neighborhood $\mathfrak{R} \subset \mathfrak{R}$ of $r_{0}$ corresponds to a domain $\Delta$ in the complex $z$-plane which contains the origin. Consider now the conformal mapping $[14,17,19,20]$

$$
z^{*}=z+\frac{e^{2 i \alpha} \varrho^{2}}{z}, \quad \varrho>0, \alpha=\text { real }
$$

in the entire $z$-plane. We assume that the disk $|z|<\varrho$ lies in $\Delta$. Outside of this disk the mapping $z \rightarrow z^{*}$ is one-to-one and regular analytic for all finite values $z$. The circumference $|z|=\varrho$ is mapped onto the rectilinear segment $\left\langle-2 \varrho e^{i \alpha}, 2 \varrho e^{i \alpha}\right\rangle$ such that the points $z_{1}=\varrho e^{i \varphi}$ and $z_{2}=\varrho e^{i(2 \alpha-\varphi)}$ go into the same point $z^{*}=2 \varrho e^{i \alpha} \cos (\varphi-\alpha)$. If we divide the circumference $|z|=\varrho$ into two arcs by drawing the diameter $z=\tau e^{i \alpha},-\varrho \leqslant \tau \leqslant \varrho$, we see that points on this circumference and symmetric to the diameter go into the same points $z^{*}$.

We are now able to define a rather radical deformation of the complex $z$-plane. We cut from it the disk $|z|<\varrho$ and identify points $z_{1}=\varrho e^{i \varphi}$ and $z_{2}=\varrho e^{i(2 \alpha-\varphi)}$, which removes all boundary points of the cut domain and makes it to a new Riemann domain. We may still use the parameter $z$ as a uniformizer on the new Riemann domain, but a function in this domain will be considered analytic only if it is an analytic function of $z^{*}=z+e^{2 i \alpha} \varrho^{2} / z$.

The deformation of the complex $z$-plane just defined determines a deformation of the Riemann surface $\mathfrak{R}$ as follows. We delete from $\mathfrak{R}$ all points in $\mathfrak{R}$ which correspond to the disk $|z|<\varrho$ in the uniformizer neighborhood of $z$ and identify points $p_{1}$ and $p_{2}$ which correspond to points $z_{1}$ and $z_{2}$ with the same value $z^{*}$. This leaves us with a new Riemann surface $\mathfrak{R}^{*}$ of the same genus as $\Re$. If $\varrho$ is small, the Riemann surface $\Re^{*}$ is near to $\mathfrak{R}$. This means that corresponding normalized Abelian integrals differ numerically arbitrarily little at corresponding points if $\varrho$ is small enough.

We now wish to give an asymptotic formula for the Szegö kernel $\Lambda^{*}(p, q)$ of the deformed surface $\mathfrak{R}^{*}$ in terms of the Szegö kernel $\Lambda(p, q)$ of the original surface $\Re$. For this purpose we introduce a canonical set of cross cuts $\left\{\mathfrak{A}_{\nu}, \mathfrak{B}_{\nu}\right\}$ for $\mathfrak{A}$ and take care that none of its loops passes through the neighborhood $\mathfrak{N}$ of the point $r_{0}$ at which we perform the variation. Under this assumption the same set may also serve as canonical cross-cut system for $\mathfrak{R}^{*}$. We construct the Szegö kernel $\Lambda^{*}(p, q)$ of $\mathfrak{R}^{*}$ by the procedure of Section III.2, using the corresponding differential of the first kind $v^{* \prime}(p)$ on $\mathfrak{R}^{*}$. Clearly, the kernel 
$\Lambda^{*}(p, q)$ so obtained will be single valued on the corresponding two-sheeted covering of $\mathfrak{R}^{*}$. In particular, we come to the fundamental conclusion that

$$
\Pi\left(p ; q_{1}, q_{2}\right)=\Lambda^{*}\left(p, q_{1}\right) \Lambda\left(p, q_{2}\right)
$$

is a differential of order 1 for $p \in \mathfrak{R}-\mathfrak{R}$ which is single valued on the residual Riemann surface. It has simple poles with the residues $\Lambda\left(q_{1}, q_{2}\right)$ at $q_{1}$ and $\Lambda^{*}\left(q_{2}, q_{1}\right)$ at $q_{2}$ if we assume that $q_{1}$ and $q_{2}$ lie also in $\mathfrak{R}-\mathfrak{R}$. Let $|z|=r(\varrho<r)$ be a fixed circumference in the uniformizer neighborhood $\mathfrak{N}$ and let $\Gamma$ be its corresponding image in $\mathfrak{R}$. We apply the residue theorem to $\Pi\left(p ; q_{1}, q_{2}\right)$ with respect to the part of $\Re$ outside of $\Gamma$. Using further the antisymmetry (III.25) of the Szegö kernel, we find

$$
\frac{1}{2 \pi i} \oint_{|z|=r} \Lambda^{*}\left(z, q_{1}\right) \Lambda\left(z, q_{2}\right) d z=\Lambda^{*}\left(q_{1}, q_{2}\right)-\Lambda\left(q_{1}, q_{2}\right)
$$

if we run over the circumference in the positive sense.

Observe that $\Lambda\left(z, q_{2}\right)$ may be developed into a convergent power series in $z$ since it is analytic on $\Re$. This is not the case for the Szegö kernel $\Lambda^{*}\left(z, q_{1}\right)$ of $\Re^{*}$ whose development proceeds in powers of $z^{*}$. Since $\Lambda^{*}(p, q)$ is a differential of order $\frac{1}{2}$, we have

$$
\Lambda^{*}\left(z, q_{1}\right)=\mathfrak{F}\left(z^{*}, q_{1}\right)\left(\frac{d z^{*}}{d z}\right)^{\frac{1}{z}}
$$

where $\mathfrak{F}\left(z^{*}, q_{1}\right)$ is a power series in $z^{*}$. Using the relation (1) between $z$ and $z^{*}$, we thus obtain

$$
\Lambda^{*}\left(z, q_{1}\right)=\mathfrak{F}\left(z, q_{1}\right)+e^{2 i \alpha} \varrho^{2}\left[\frac{\mathfrak{F}^{\prime}\left(z, q_{1}\right)}{z}-\frac{\mathfrak{F}\left(z, q_{1}\right)}{2 z^{2}}\right]+O\left(\varrho^{4}\right)
$$

Inserting (5) into (3), we may now apply the residue theorem with respect to $|z|<r$ since $\mathfrak{F}\left(z, q_{1}\right)$ is analytic there. We find the asymptotic formula

$$
\Lambda^{*}\left(q_{1}, q_{2}\right)-\Lambda\left(q_{1}, q_{2}\right)=\frac{1}{2} e^{2 i \alpha} \varrho^{2}\left[\mathfrak{F}^{\prime}\left(0, q_{1}\right) \Lambda\left(0, q_{2}\right)-\Lambda^{\prime}\left(0, q_{2}\right) \mathfrak{F}\left(0, q_{1}\right)\right]+O\left(\varrho^{4}\right) .
$$

Since $\Lambda^{*}\left(z, q_{1}\right)$ depends on $\varrho$ so does $\mathfrak{F}\left(z^{*}, q_{1}\right)$. But it is evident that this analytic function remains bounded as $\varrho \rightarrow 0$; we therefore infer from $(6)$ that $\Lambda^{*}\left(q_{1}, q_{2}\right)-\Lambda\left(q_{1}, q_{2}\right)=O\left(\varrho^{2}\right)$ uniformly in each closed region in $\mathfrak{R}$ which does not contain the point $r_{0}$ at which we deform the surface. From this fact and (5) we can obtain

$$
\Lambda\left(z, q_{1}\right)-\mathfrak{F}\left(z, q_{1}\right)=O\left(\varrho^{2}\right) \text { for }|z|=r .
$$

Since the left hand of $(7)$ is analytic for $|z|<r$, we infer the same asymptotic formula for all $|z|<r$ and, in particular, 


$$
\Lambda\left(0, q_{1}\right)=\mathfrak{F}\left(0, q_{1}\right)+O\left(\varrho^{2}\right), \quad \Lambda^{\prime}\left(0, q_{1}\right)=\mathfrak{F}^{\prime}\left(0, q_{1}\right)+O\left(\varrho^{2}\right)
$$

Hence, finally (6) takes the symmetric form

$$
\Lambda^{*}\left(q_{1}, q_{2}\right)-\Lambda\left(q_{1}, q_{2}\right)=\frac{1}{2} e^{2 i \alpha} \varrho^{2}\left[\Lambda^{\prime}\left(0, q_{1}\right) \Lambda\left(0, q_{2}\right)-\Lambda^{\prime}\left(0, q_{2}\right) \Lambda\left(0, q_{1}\right)\right]+O\left(\varrho^{4}\right) .
$$

This formula allows an asymptotic estimate of the new Szegö kernel $\Lambda^{*}(p, q)$ in terms of the original known Szegö kernel $\Lambda(p, q)$ and its first derivative. The error term can be estimated uniformly in each closed region of $\mathfrak{M}$ which does not contain $r_{0}$.

We summarize the result of this section as follows. If we perform a variation of $\mathfrak{R}$ at a point $r_{0}$, which in terms of the local uniformizer $z$ has the form (1), we have

$$
\delta \Lambda(p, q)=\frac{1}{2} e^{2 i \alpha} \varrho^{2}\left[\Lambda^{\prime}\left(r_{0}, p\right) \Lambda\left(r_{0}, q\right)-\Lambda\left(r_{0}, p\right) \Lambda^{\prime}\left(r_{0}, q\right)\right]
$$

where

$$
\Lambda^{\prime}\left(r_{0}, q\right)=\left.\frac{\partial}{\partial z} \Lambda(r, q)\right|_{z=0}
$$

In the case of plane domains the variational formula for the Szegö kernel was derived in [16].

2. The variational formula for the Szegö kernel $\Lambda(p, q)$ leads us to the interesting combination

$$
H_{\mathbf{0}}(r ; p, q)=\Lambda^{\prime}(r, p) \Lambda(r, q)-\Lambda(r, p) \Lambda^{\prime}(r, q)
$$

It is antisymmetric in $p$ and $q$ and clearly a differential of order $\frac{1}{2}$ in each variable. It is easily verified that it is a quadratic differential in $r$; indeed, we can write

$$
H_{0}(r ; p, q)=\Lambda(r, q)^{2} \frac{\partial}{\partial r}\left(\frac{\Lambda(r, p)}{\Lambda(r, q)}\right)
$$

which displays clearly the covariant character of $H_{0}$.

It is clear that $H_{0}$ in dependence on $r$ has two singularities, namely at points $p$ and $q$. If we introduce at $p$ a local uniformizer $\zeta(r)$ such that $\zeta(p)=0$, we find by (III.24)

$$
H_{0}(r ; p, q)=-\frac{1}{\zeta^{2}} \Lambda(p, q)-\frac{2}{\zeta} \Lambda^{\prime}(p, q)+\text { regular terms }
$$

as the series development near $p$.

We construct next the expression

$$
H_{1}(r ; p, q)=-\Lambda(p, q)\left\{\Lambda^{\prime}(r, p) \Lambda(r, q)-\Lambda(r, p) \Lambda^{\prime}(r, q)\right\} .
$$

It is now symmetric in $p$ and $q$ and is a quadratic differential in $r$, a linear differential in $p$ and $q$. We have thus succeeded in constructing a differential of integer order in all three 
variables. For $r$ near $p$, we have by (13) the development in terms of the local uniformizer $\zeta(r)$ :

$$
H_{1}(r ; p, q)=\frac{1}{\zeta^{2}} \Lambda(p, q)^{2}+\frac{1}{\zeta} 2 \Lambda^{\prime}(p, q) \Lambda(p, q)+\ldots
$$

We observe that the simpler expression $\Lambda^{2}(r, p) \Lambda^{2}(r, q)$ is also symmetric in $p$ and $q$, is a quadratic differential in $r$, and a linear differential in $p$ and $q$ and has for $r$ near $q$ precisely the same principal part (15). Indeed, by (III.24) we have for $\Lambda(r, p)$ the series development

$$
\Lambda(r, p)=\frac{1}{\zeta}+\alpha_{1} \zeta+\alpha_{3} \zeta^{3}+\ldots
$$

since it is antisymmetric in $r$ and $p$ and must be an odd power series. Thus we can assert that

$$
H_{1}(r ; p, q)-\Lambda^{2}(r, p) \Lambda^{2}(r, q)=H_{2}(r ; p, q)
$$

is symmetric in $p, q$, a linear differential in each of them, a quadratic differential in $r$, and regular analytic on $\mathfrak{R}$ in all its variables.

To understand more clearly the significance of this term

$$
-\Lambda(p, q)\left\{\Lambda^{\prime}(r, p) \Lambda(r, q)-\Lambda(r, p) \Lambda^{\prime}(r, q)\right\}-\Lambda^{2}(r, p) \Lambda^{2}(r, q)
$$

we shall identify it as the limit case of a more general expression which involves four variables but is of particularly simple structure. We define

$$
\begin{aligned}
N(r, s ; p, q)=\Lambda(r, s) \Lambda(p, q)\{\Lambda(r, p) \Lambda(s, q) & -\Lambda(r, q) \Lambda(s, p)\} \\
& +\Lambda(r, p) \Lambda(r, q) \Lambda(s, p) \Lambda(s, q)
\end{aligned}
$$

This is a linear differential in all four variables. It has the symmetries

$$
N(r, s ; p, q)=N(s, r ; p, q)=N(r, s ; q, p)=N(p, q ; r, s)=N(p, s ; r, q)
$$

We easily verify that it remains finite in each variable on $\Re$. Hence we can express it in terms of the Abelian differentials of the first kind and obtain the multilinear representation

$$
N(r, s ; p, q)=\sum_{i, k, i, m=1}^{g} c_{i k i m} w_{i}^{\prime}(r) w_{k}^{\prime}(s) w_{l}^{\prime}(p) w_{m}^{\prime}(q)
$$

The symmetries (20) express themselves in terms of the coefficients as

$$
c_{i k l m}=c_{k i l m}=c_{i k m l}=c_{l m i k}=c_{l k i m} \text {. }
$$

The coefficients are thus completely symmetric in all four indices.

15-662945 Acta mathematica. 115. Imprimé le 11 mars 1966. 
Let us write the identity (21) by means of the definition (19) in the form

$$
\begin{aligned}
& -\Lambda(r, s) \Lambda(p, q)\{\Lambda(r, p) \Lambda(s, q)-\Lambda(r, q) \Lambda(s, p)\} \\
& =\Lambda(r, p) \Lambda(r, q) \Lambda(s, p) \Lambda(s, q)-\sum_{i, k, l, m=1}^{g} c_{i k l m} w_{i}^{\prime}(r) w_{k}^{\prime}(s) w_{l}^{\prime}(p) w_{m}^{\prime}(q) .
\end{aligned}
$$

Pass here to the limit $s=r$ and find $H_{1}(r ; p, q)$ :

$$
\begin{aligned}
-\Lambda(p, q)\left\{\Lambda^{\prime}(r, p)\right. & \left.\Lambda(r, q)-\Lambda^{\prime}(r, q) \Lambda(r, p)\right\} \\
& =\Lambda(r, p)^{2} \Lambda(r, q)^{2}-\sum_{i, k, i, m=1}^{g} c_{i k l m} w_{i}^{\prime}(r) w_{k}^{\prime}(r) w_{l}^{\prime}(p) w_{m}^{\prime}(q) .
\end{aligned}
$$

We have thus found a simple and highly symmetric expression for the important variational terms (11), (14), and (18) by means of differentials of the first kind. The coefficient set $c_{i k l m}$ is a set of possible moduli for the surface $\Re$. We shall show its importance in the general theory of moduli on a Riemann surface.

3. We have derived in Section 1 a variational formula for the Szegö kernel and obtained in Section 2 remarkable identities for the variational expressions which will facilitate its applications. We wish to show now to what use the entire variational theory can be put.

We return to the identity (II.9) which must be fulfilled by the Szegö kernel

$$
\Lambda(p, q)^{2}=L(p, q)+\sum_{i, k=1}^{g} a_{i k} w_{i}^{\prime}(p) w_{k}^{\prime}(q)
$$

The coefficient matrix $\left(\left(a_{i k}\right)\right)$ is symmetric and uniquely determined by the Riemann surface $\mathfrak{R}$ and the two-sheeted covering on which $\Lambda(p, q)$ is single valued. The $a_{i k}$ may thus be considered as a set of moduli for $\Re$. A very similar symmetric $g \times g$ matrix of moduli is given by the period matrix $\left(\left(P_{i k}\right)\right)$ of the integrals of the first kind as defined in (III.16). This matrix has been extensively studied and its importance in the moduli problem is well known. The question arises whether the two matrices $\left(\left(a_{i k}\right)\right)$ and $\left(\left(P_{i k}\right)\right)$ stand in any simple relation.

Let $\Gamma_{1}$ and $\Gamma_{2}$ be two closed curves on $\Re$. Integrating the identity (25) with $p \in \Gamma_{1}$, $q \in \Gamma_{2}$, we obtain the equation

$$
\int_{\Gamma_{1}} \int_{\Gamma_{\mathbf{2}}} \Lambda(p, q)^{2} d p d q=\int_{\Gamma_{1}} \int_{\Gamma_{\mathbf{z}}} L(p, q) d p d q+\sum_{i, k=1}^{g} a_{i k} \int_{\Gamma_{\mathbf{1}}} d w_{i} \int_{\Gamma_{\mathbf{3}}} d w_{k}
$$

By definition (III: 8 ) of the $L$-kernel and in view of (I: 15$)$ we have

$$
\int_{\Gamma} L(p, q) d p=\int_{\Gamma} \frac{\partial^{2} w(p ; q, s)}{\partial p \partial q} d p=\frac{\partial}{\partial q} \int_{\Gamma} d w(p ; q, s) .
$$


Because of the normalization of the Abelian integrals of the third kind we then obtain

$$
\int_{\mathfrak{A}_{\alpha}} L(p, q) d p=0, \quad \int_{\mathfrak{P}_{\alpha}} L(p, q) d p=2 \pi i w_{\alpha}^{\prime}(q),
$$

and consequently

$$
\begin{aligned}
& \int_{\mathfrak{A}_{\alpha}} \int_{\mathscr{A}_{\beta}} L(p, q) d p d q=\int_{\mathfrak{P}_{\alpha}} \int_{\mathscr{N}_{\beta}} L(p, q) d p d q=0, \\
& \int_{\mathfrak{P}_{\alpha}} \int_{\mathfrak{P}_{\beta}} L(p, q) d p d q=2 \pi i \delta_{\alpha \beta}
\end{aligned}
$$

and

$$
\int_{\mathfrak{B}_{\varkappa}} \int_{\mathfrak{F}_{\beta}} L(p, q) d p d q=2 \pi i P_{\alpha \beta}
$$

Observe the asymmetry of the integrals extended over $\mathfrak{A}_{\alpha}$ and $\mathfrak{P}_{\alpha}$. It is due to the fact that the integral is in this case improper and therefore, in spite of the symmetry of its kernel, takes different values for a different order of integration.

The equations (26), (29), $\left(29^{\prime}\right)$ and $\left(29^{\prime \prime}\right)$ lead to the period formulas

$$
\begin{aligned}
& \int_{\mathfrak{X}_{\alpha}} \int_{\mathfrak{A}_{\beta}} \Lambda(p, q)^{2} d p d q=a_{\alpha \beta}, \\
& \int_{\mathfrak{B}_{\alpha}} \int_{\mathfrak{N}_{\beta}} \Lambda(p, q)^{2} d p d q=\sum_{k=1}^{g} \boldsymbol{P}_{\alpha k} a_{k \beta}, \\
& \int_{\mathfrak{A}_{\alpha}} \int_{\mathfrak{F}_{\beta}} \Lambda(p, q)^{2} d p d q=2 \pi i \delta_{\alpha \beta}+\sum_{k=1}^{g} a_{\alpha k} P_{k \beta}, \\
& \int_{\mathfrak{B}_{\alpha}} \int_{\mathfrak{B}_{\beta}} \Lambda(p, q)^{2} d p d q=2 \pi i P_{\alpha \beta}+\sum_{i, k=1}^{g} P_{\alpha 1} a_{i k} P_{k \beta} .
\end{aligned}
$$

The symmetric matrix $\left(\left(a_{\alpha \beta}\right)\right)$ of coefficients in (25) has thus been identified as the period matrix of $\Lambda(p, q)^{2}$ with respect to the cycles $\mathfrak{A}_{\alpha}$ :

$$
A=\left(\left(a_{\alpha \beta}\right)\right)=\left(\left(\int_{\mathfrak{A}_{\alpha}} \int_{\mathfrak{A}_{\beta}} \Lambda(p, q)^{2} d p d q\right)\right) .
$$

It is therefore very analogous to the Riemann matrix $\left(\left(P_{\alpha \beta}\right)\right)$ which is the period matrix of the $L$-kernel with respect to the cycles $\mathfrak{B}_{\alpha}$ :

$$
P=\left(\left(2 \pi i P_{\alpha \beta}\right)\right)=\left(\left(\int_{\mathfrak{B}_{\alpha}} \int_{\mathfrak{B}_{\beta}} L(p, q) d p d q\right)\right) .
$$


We define next the matrices

and

$$
\begin{aligned}
& K=\left(\left(k_{\alpha \beta}\right)\right)=\left(\left(\int_{\mathfrak{B}_{\alpha}} \int_{\mathfrak{X}_{\beta}} \Lambda(p, q)^{2} d p d q\right)\right) \\
& \hat{K}=\left(\left(\hat{k}_{\alpha \beta}\right)\right)=\left(\left(\int_{\mathfrak{A}_{\alpha}} \int_{\mathfrak{P}_{\beta}} \Lambda(p, q)^{2} d p d q\right)\right) .
\end{aligned}
$$

While $A$ and $P$ are symmetric matrices, $K$ and $R$ are not. By $\left(30^{\prime}\right)$ and (30") we have

$$
\hat{K}=2 \pi i I+K^{T}
$$

where $I$ is the unit matrix and $K^{T}$ is the transposed matrix of $K$. We can now bring the equations $\left(30^{\prime}\right)$ and $\left(30^{\prime \prime \prime}\right)$ into matrix form if we also define the period matrix of $\Lambda(p, q)^{2}$ with respect to the $\mathfrak{B}$-cycles:

$$
B=\left(\left(b_{\alpha \beta}\right)\right)=\left(\left(\int_{\mathscr{B}_{\alpha}} \int_{\mathscr{B}_{\beta}} \Lambda(p, q)^{2} d p d q\right)\right) .
$$

We find

$$
K=\frac{1}{2 \pi i} P \cdot A ; \quad B=P-\frac{1}{4 \pi^{2}} P A P .
$$

We can condense the matrix relations into one single equation if we introduce the symmetric matrix

$$
S=\left(\begin{array}{ll}
A & K^{T} \\
K & B
\end{array}\right)
$$

which depends only on the periods of $\Lambda^{2}(p, q)$ and the matrix

$$
\Sigma=\left(\begin{array}{cc}
I & 0 \\
\frac{1}{2 \pi i} P & I
\end{array}\right)
$$

which depends only on the periods of $L(p, q)$. The equations (35) can then be combined in the matrix equation

$$
S=\Sigma\left(\begin{array}{ll}
A & 0 \\
0 & P
\end{array}\right) \Sigma^{T}
$$

4. After these formal considerations we are now ready to study the dependence of the various period matrices upon the Riemann surface $\mathfrak{R}$ for which they are defined. By virtue of the identities (35) or (38) it is sufficient to know how the matrices $P$ and $A$ change under a deformation of the surface $\mathfrak{R}$ in order to compute the change of the remaining periods under the same variation. 
It is known that under a variation of $\Re$ at $r_{0}$ according to (1) we have the asymptotic formula $[14,20]$

$$
\delta P_{\mu \nu}=-2 \pi i e^{2 i \alpha} \varrho^{2} w_{\mu}^{\prime}\left(r_{0}\right) w_{\nu}^{\prime}\left(r_{0}\right)
$$

To find the variation of the matrix $A$ we have to use the identity (30) and the known variational formula for the Szegö kernel. By use of (10) and (14) we find

$$
\delta a_{\mu \nu}=-e^{2 \ell \alpha} \varrho^{2} \int_{\mathfrak{A}_{\mu}} \int_{\mathfrak{A}_{\nu}} H_{1}\left(r_{0} ; p, q\right) d p d q .
$$

We simplify considerably by representing $H_{1}\left(r_{0} ; p, q\right)$ by means of (24). Indeed,

$$
\delta a_{\mu \nu}=-e^{21 \alpha} Q^{2}\left\{\int_{\mathfrak{U}_{\mu}} \Lambda\left(r_{0}, p\right)^{2} d p \int_{\mathfrak{X}_{\nu}} \Lambda\left(r_{0}, q\right)^{2} d q-\sum_{j, k=1}^{g} c_{j k \mu \nu} w_{j}^{\prime}\left(r_{0}\right) w_{k}^{\prime}\left(r_{0}\right)\right\}
$$

We reduce the formulas further by use of (25) and (28) which yield

$$
\int_{\mathfrak{R}_{\mu}} \Lambda\left(r_{0}, p\right)^{2} d p=\sum_{j=1}^{g} a_{j \mu} w_{j}^{\prime}\left(r_{0}\right)
$$

We therefore arrive at the final result:

$$
\delta a_{\mu \nu}=e^{2 l \alpha} \varrho^{2}\left\{\sum_{j, k=1}^{g} c_{j k \mu \nu} w_{j}^{\prime}\left(r_{0}\right) w_{k}^{\prime}\left(r_{0}\right)-\sum_{j, k=1}^{g} a_{j \mu} a_{k \nu} w_{j}^{\prime}\left(r_{0}\right) w_{k}^{\prime}\left(r_{0}\right)\right\}
$$

If we combine the variational formulas (39) and (43), we obtain the elegant equation

$$
2 \pi i \delta a_{\mu \nu}=\sum_{j, k=1}^{g}\left(a_{j \mu} a_{k \nu}-c_{j k \mu \nu}\right) \delta P_{j k}
$$

Let us introduce a set of moduli $m_{\alpha}$ of which the $P_{j k}$ and $a_{j k}$ are real analytic functions. We then find

$$
2 \pi i \frac{\partial a_{\mu \nu}}{\partial m_{\alpha}}=\sum_{j, k=1}^{g}\left(a_{j \mu} a_{k \nu}-c_{i k \mu \nu}\right) \frac{\partial P_{j k}}{\partial m_{\alpha}}
$$

Observe that because of (22) we have

This implies $\quad \sum_{\mu, \nu=1}^{g}\left(\frac{\partial a_{\mu \nu}}{\partial m_{\alpha}} \frac{\partial P_{\mu \nu}}{\partial m_{\beta}}-\frac{\partial a_{\mu \nu}}{\partial m_{\beta}} \frac{\partial P_{\mu \nu}}{\partial m_{\alpha}}\right)=0$

$$
a_{j \mu} a_{k p}-c_{j k \mu \nu}=a_{\mu j} a_{v k}-c_{\mu \nu j k}
$$

for any pair of indices $\alpha$ and $\beta$. But (47) is the well-known integrability condition which guarantees that the integral 


$$
\int \sum_{\mu, v=1}^{\rho} a_{\mu \nu} d P_{\mu \nu}=J
$$

is unchanged under continuous deformation of the path in the space of moduli provided the end points are kept fixed. Thus we can define a function $J\left(m_{\alpha}\right)$ in the space of moduli such that

$$
d J=\sum_{\mu, \nu=1}^{o} a_{\mu \nu} d P_{\mu \nu}
$$

These identities and theorems illustrate the value and the significance of the variational formulas for the various differentials on a Riemann surface $\mathfrak{R}$. The amount of new identities and suggestive relations involving the Szegö kernel show the usefulness of the new concept for the general theory of Abelian integrals.

5. We introduced the coefficient scheme $c_{j k \mu \nu}$ by the definition (21), and we may also characterize them in view of $(44)$ as differential coefficients of the matrix $\left(\left(a_{\mu \nu}\right)\right)$ with respect to the matrix $\left(\left(P_{j k}\right)\right)$. Another interesting role for this set of coefficients can be deduced from identity (23) if we specialize the point $p$ in this formula to be a point $q_{\nu}$ associated to $q$ according to the relation

In this case, (23) reduces to

$$
\Lambda\left(q_{\nu}, q\right)=0
$$

$$
\sum_{i, k, l, m=1}^{g} c_{i k l m} w_{i}^{\prime}(r) w_{k}^{\prime}(s) w_{l}^{\prime}\left(q_{\nu}\right) w_{m}^{\prime}(q)=\Lambda\left(r, q_{\nu}\right) \Lambda(r, q) \Lambda\left(s, q_{\nu}\right) \Lambda(s, q) .
$$

On the other hand, we showed in Section III.3 that $v_{\nu}^{\prime}(p)=\Lambda(p, q) \Lambda\left(p, q_{\nu}\right)$ is a differential of the first kind if $q$ and $q_{v}$ are associated points. We may express each $v_{v}^{\prime}(p)$ in terms of the canonical basis for such differentials and write

$$
v_{\nu}^{\prime}(p)=\Lambda(p, q) \Lambda\left(p, q_{\nu}\right)=\sum_{\varrho=1}^{g} c_{v e}(q) w_{\varrho}^{\prime}(p) .
$$

With the coefficient matrix $\left(\left(c_{v e}(q)\right)\right)$ so defined and in view of the linear independence of all $w_{\nu}^{\prime}(p)$ we derive from $(50)$ the identity

$$
\sum_{l, m=1}^{g} c_{i k l m} w_{l}^{\prime}\left(q_{v}\right) w_{m}^{\prime}(q)=c_{p l}(q) c_{p k}(q)
$$

We can eliminate from this identity the $c_{p \rho}(q)$ entirely and bring (52) into the form

$$
\sum_{l, m, \lambda_{,}, \mu=1}^{g}\left(c_{i k l m} c_{i k \lambda \mu}-c_{i i l m} c_{k \kappa \lambda \mu}\right) w_{l}^{\prime}\left(q_{v}\right) w_{\lambda}^{\prime}\left(q_{v}\right) w_{m}^{\prime}(q) w_{\mu}^{\prime}(q)=0 .
$$


It is convenient to introduce the bilinear forms of quadratic differentials

$$
B_{i k}(p, q)=\sum_{l, m, \hat{\lambda}, \mu=1}^{g}\left(c_{i k l m} c_{i k \lambda \mu}-c_{i i l m} c_{k k \lambda \mu}\right) w_{l}^{\prime}(p) w_{\lambda}^{\prime}(p) w_{m}^{\prime}(q) w_{\mu}^{\prime}(q)
$$

which is based on the coefficient scheme $c_{i k l m}$. We then see by (53) that

$$
B_{i k}\left(q_{\nu}, q\right)=0, \quad 1 \leqslant i<k \leqslant g
$$

for any pair of associated points $q_{\nu}, q$ on $\Re$.

We may also express the property (55) by writing

$$
B_{i k}(p, q)=\Lambda(p, q) T_{i k}(p, q)
$$

where the kernels $T_{i k}(p, q)$ are antisymmetric in $p$ and $q$ and 3/2-order differentials in each variable. To represent the $T_{i k}(p, q)$ in a simple manner, we have to investigate the class of all regular $3 / 2$-order differentials on $\mathfrak{R}$ which are single valued on the same twosheeted covering of $\Re$ as $\Lambda(p, q)$.

We easily see that

$$
T(p)=\Lambda(p, q)\left\{\sum_{\nu=1}^{g} C_{\nu} w^{\prime}\left(p ; q_{\nu}, q_{0}\right)+\sum_{\nu=1}^{g} c_{\nu} w_{\nu}^{\prime}(p)\right\}
$$

will be the most general, regular $3 / 2$-order differential if the coefficients $C_{\nu}$ and $c_{\nu}$ satisfy the two linear, homogeneous conditions

$$
\sum_{\nu=1}^{g} C_{\nu}=0, \quad \sum_{\nu=1}^{g} C_{\nu} w^{\prime}\left(q ; q_{\nu}, q_{0}\right)+\sum_{\nu=1}^{g} c_{\nu} w_{\nu}^{\prime}(q)=0 .
$$

The point $q_{0} \in \Re$ can be chosen arbitrarily except for being different from $q$. There are precisely $2(g-1)$ linearly independent regular $3 / 2$-order differentials on $\mathfrak{R}$. We choose a basis $T_{\alpha}(p)(\alpha=1, \ldots 2(g-1))$ of such differentials and can then write

$$
T_{i k}(p, q)=\sum_{\alpha, \beta=1}^{2 g-2} d_{t k, \alpha \beta} T_{\alpha}(p) T_{\beta}(q)
$$

with $d_{i k, \alpha \beta}$ being antisymmetric in the last pair of indices.

Observe now that there are $3 g-3$ linearly independent quadratic differentials. Hence the most general symmetric bilinear form of quadratic differentials depends on $\left(\begin{array}{c}3 g-2 \\ 2\end{array}\right)$ independent coefficients. On the other hand, the antisymmetric bilinear forms of differentials of order $3 / 2$ depend only upon $\left(\begin{array}{c}2 g-2 \\ 2\end{array}\right)$ independent parameters. Thus, each of the coefficient sets of the forms $B_{i k}(p, q)$ has to satisfy a large number of constraints. 
These set up numerous conditions in the matrix $\left(\left(c_{i k l m}\right)\right)$ which may be interpreted as differential relations for the $\left(\left(a_{t k}\right)\right)$ in their dependence on the $\left(\left(P_{l m}\right)\right)$.

The significance of the variational formula for the Szegö kernel in the problem of the moduli of a Riemann surface and the Teichmüller spaces is evident. These problems have been treated extensively and successfully in recent years $[1,6,7]$. We hope to be able to contribute to these questions by the present developments and techniques.

\section{Bibliography}

[1]. Ahlfors, L. V., Teichmüller spaces. Proc. Intern. Congr. Math., Stockholm 1962, 3-9.

[2]. Appenl, P. \& Gounsat, E., Théorie des fonctions algébriques, t. II. Paris 1930.

[3]. Bergmax, S., Über die Entwicklung der harmonischen Funktionen der Ebene und des Raumes nach Orthogonalfunktionen. Math. Ann., 86 (1922), 238-271.

[4]. - The kernel function and conformal mapping. Amer. Math. Soc. Survey, New York 1950.

[5]. Bergman, S. \& Schiffer, M., Kernel functions and conformal mapping. Compositio Math., 8 (1951), 205-249.

[6]. Bers, L., Spaces of Riemann surfaces. Proc. Intern. Congr. Math., Edinburgh 1958, 349-361.

[7]. - - Automorphic forms and general Teichmüller spaces. Proc. Conf. Complex Anal., Minneapolis 1964, 109-113.

[8]. Courant, R., Dirichlet's principle, conformal mapping and minimal surfaces. Appendix by M. Schiffer. New York 1950.

[9]. Garabedran, P. R., Schwarz's lemma and the Szegö kernel function. Trans. Amer. Math. Soc., 67 (1949), 1-35.

[10]. Hensel, K. \& Landsberg, G., Theorie der algebraischen Funktionen einer Variabeln. Leipzig 1902.

[11]. Hule, E., Remarks on a paper by Zeev Nehari. Bull. Amer. Math.Soc., 55 (1949), 552-553.

[12]. KrazER, A., Lehrbuch der Thetafunktionen. Leipzig 1903.

[13]. Nehari, Z., The Schwarzian derivative and schlicht functions. Bull. Amer. Math. Soc., 55 (1949), 545-551.

[14]. SCHFFER, M., Hadamard's formula and variation of domain functions. Amer.J. Math., 68 (1946), 417-448.

[15]. - Faber polynomials in the theory of univalent functions. Bull. Amer. Math. Soc., 54 (1948), 503-517.

[16]. - Various types of orthogonalization. Duke Math. J., 17 (1950), 329-366.

[17]. - Variational methods in the theory of Rieman surfaces. Contributions to the theory of Riemann surfaces, Princeton 1953, 15-30.

[18]. Schiffer, M. \& Hawley, N. S., Connections and conformal mapping. Acta Math., 107 (1962), 175-274.

[19]. Schiffer, M. \& Spencer, D. C., A variational calculus for Riemann surfaces. Ann. Acad. Scient. Fenn. Ser. A I, 93, Helsinki 1951.

[20]. - Functionals of finite Riemann surfaces. Princeton 1954.

[21]. Szeqö, G., Über orthogonale Polynome, die zu einer gegebenen Kurve der komplexen Ebene gehören. Math. Z., 9 (1921), 218-270.

[22]. WEYL, H., Die Idee der Riemannschen Fläche. 3. Aufl., Stuttgart 1955.

Received July 31, 1965 